Pure and Applied Mathematics Quarterly

Volume 4, Number 4

(Special Issue: In honor of

Jean-Pierre Serre, Part 1 of 2)

$1233-1278,2008$

\title{
Diagonal Subschemes and Vector Bundles
}

\section{Piotr Pragacz, Vasudevan Srinivas and Vishwambhar Pati}

\author{
To Professor Jean-Pierre Serre on his 80th birthday
}

Abstract: We study when a smooth variety $X$, embedded diagonally in its Cartesian square, is the zero scheme of a section of a vector bundle of rank $\operatorname{dim}(X)$ on $X \times X$. We call this the diagonal property (D). It was known that it holds for all flag manifolds $\mathrm{SL}_{n} / P$.

We consider mainly the cases of proper smooth varieties, and the analogous problems for smooth manifolds ("the topological case").

Our main new observation in the case of proper varieties is a relation between (D) and cohomologically trivial line bundles on $X$, obtained by a variation of Serre's classic argument relating rank 2 vector bundles and codimension 2 subschemes, combined with Serre duality. Based on this, we have several detailed results on surfaces, and some results in higher dimensions.

For smooth affine varieties, we observe that for an affine algebraic group over an algebraically closed field, the diagonal is in fact a complete intersection; thus (D) holds, using the trivial bundle. We conjecture the existence of smooth affine complex varieties for which (D) fails; this leads to an interesting question on projective modules.

The arguments in the topological case have a different flavour, with arguments from homotopy theory, topological K-theory, index theory etc. There are 3 variants of the diagonal problem, depending on the type of vector bundle we want (arbitrary, oriented or complex). We obtain a homotopy theoretic reformulation of the diagonal property as an extension problem for a certain homotopy class of maps. We also have detailed results in several cases: spheres, odd dimensional complex projective quadric hypersurfaces, and manifolds of even dimension $\leq 6$ with an almost complex structure.

Key words and Phrases. Diagonal, vector bundle, cohomologically trivial line bundle.

Received August 19, 2006.

1991 Mathematics Subject Classification. 14F05,14F45,57R22.

Pragacz's research supported by the Humboldt Stiftung during his stay at the MPIM in Bonn in the period May-July 2006. 


\section{CONTEnTs}

1. Introduction

2. Preliminaries on (D)

2.1. Some general remarks 1238

2.2. (D) for proper smooth varieties 1238

3. Main results for surfaces 1243

3.1. (D) within a birational class 1243

3.2. Ruled surfaces 1246

3.3. Surfaces of Kodaira dimension $0 \quad 1247$

3.4. Elliptic surfaces 1250

3.5. Some surfaces for which (D) fails 1251

4. Higher dimensional varieties 1253

4.1. Varieties with Picard group $\mathbb{Z} \quad 1253$

4.2. (D) and the Point Property 1254

5. The affine case 1257

6. The Topological Diagonal Property 1258

References $\quad 1276$

\section{INTRODUCTION}

Diagonal subschemes are important in many questions of intersection theory. Apart from classical "reduction to the diagonal" (cf., e.g., [32] or [9]), it was shown in [28], Sect.5 that knowing the fundamental class of the diagonal of a variety, is an important step towards computing the fundamental classes of all subschemes of this variety (see also [29], [12]).

A good resolution of the structure sheaf of the diagonal over the structure sheaf of $X \times X$ has been used to give a description of the derived category $D(X)$ of $X$ [19], and has proved useful in studying algebraic $K$-theory of homogeneous spaces and their twisted forms (cf. [21], [4]).

We recall an interesting case, where the diagonal is described in a suitable fashion, leading to a resolution of its structure sheaf by a Koszul complex. Let $G=G_{r}(V)$ be the Grassmannian parametrizing all $r$-subspaces of a vector space 
$V$ (so, in particular, for $r=1$, we consider projective spaces). The Grassmannian is endowed with the "tautological" sequence of vector bundles

$$
0 \rightarrow S \rightarrow V_{G} \rightarrow Q \rightarrow 0,
$$

where $\operatorname{rank}(S)=r$. Let $G_{1}=G_{2}=G$ and use analogous notation for the tautological vector bundles on $G_{i}, i=1,2$. Denote by

$$
p_{1}, p_{2}: G_{1} \times G_{2} \rightarrow G
$$

the two projections. Then the diagonal of $G$ is the zero scheme of the section $s$ of the bundle

$$
\underline{\operatorname{Hom}}\left(p_{1}^{*} S_{1}, p_{2}^{*} Q_{2}\right)
$$

of rank equal to $\operatorname{dim}(G)$ on $G_{1} \times G_{2}$, where $s$ is induced by the following vector bundle homomorphism:

$$
p_{1}^{*} S_{1} \rightarrow p_{1}^{*} V_{G_{1}}=V_{G_{1} \times G_{2}}=p_{2}^{*} V_{G_{2}} \rightarrow p_{2}^{*} Q_{2} .
$$

(This was surely observed independently by so many people that the "paternity" is impossible to detect. ${ }^{1}$ )

Let $X$ be a smooth variety. Denote by $\Delta \subset X \times X$ the diagonal subscheme, that is, the image of the diagonal embedding

$$
\delta: X \hookrightarrow X \times X,
$$

given by $\delta(x)=(x, x)$.

We are interested in when the following diagonal property holds:

"There exists a vector bundle $\mathcal{E}$ of rank equal to $\operatorname{dim}(X)$ on $X \times X$ and a section $s$ of $\mathcal{E}$, such that $\Delta$ is the zero scheme of $s . "$

In the following, we shall use abbreviation "(D)" for the diagonal property.

Observe that if $X_{1}$ and $X_{2}$ satisfy (D), then it also holds for $X_{1} \times X_{2}$. Moreover, (D) is obviously valid for curves. We noted that it holds for any Grassmannian; it also holds for flag varieties of the form $S L_{n} / P$ over any field (cf. [10], [11]). In fact, in this last case, (D) was a starting point for the development of the Lascoux-Schützenberger theory of Schubert polynomials for $S L_{n}$ [20] (see also [10], [11]). The question:

"Do the flag varieties for other classical groups have (D)?"

arose in discussions of the first author with William Fulton while writing up [11] at the University of Chicago in 1996.

\footnotetext{
1 - even by analyzing the DNA.
} 
In the present paper, we investigate this property mainly for surfaces, and have some results in the higher dimensional case. We use an argument arising from the fundamental Serre construction relating codimension 2 subschemes and vector bundles of rank 2 (cf. [31]), combined with Serre duality, to relate (D) with the existence or absence of cohomologically trivial line bundles.

In the surface case, this leads to the following result, summarizing several conclusions.

Theorem 1. Let $X$ be a smooth projective surface over an algebraically closed field.

(a) There exists a birational proper morphism $f: Y \rightarrow X$ such that $Y$ is smooth, and satisfies (D) (see Theorem 5 below).

(b) If $Y \rightarrow X$ is a birational morphism, $X$ satisfies $(D)$, and $\operatorname{Pic}(X)$ is finitely generated, then $Y$ satisfies (D).

(c) Suppose $X$ is birational to one of the following: a ruled or an abelian surface, or a K3 surface with two disjoint rational curves, or an elliptic fibration with a section, or a product of 2 curves, or a complex Enriques or hyperelliptic surface. Then $X$ satisfies (D).

(d) Suppose $\operatorname{Pic}(X)=\mathbb{Z}$, such that the ample generator of $\operatorname{Pic}(X)$ has a nonzero section, and $X$ satisfies $(D)$. Then $X \cong \mathbb{P}^{2}$. In particular, (D) fails for general algebraic K3 surfaces, or for general hypersurfaces $X \subset$ $\mathbb{P}^{3}$ of degree $\geq 4$.

For higher dimensional varieties, the general result we have is the following.

Theorem 2. Let $X$ be a smooth projective variety with $\operatorname{Pic}(X)=\mathbb{Z}$, such that the ample generator $\mathcal{O}_{X}(1)$ has a nonzero section. Suppose $X$ has (D). Then $\omega_{X} \cong \mathcal{O}_{X}(-r)$ for some $r \geq 2$, i.e., $X$ is a Fano variety of index $\geq 2$.

On the other hand, (D) fails for a smooth projective quadric of any odd dimension $\geq 3$. Curiously, our proof of this for quadrics of dimension $\geq 5$ is by reducing to the case of quadrics over the complex numbers, and using the topological results below.

We have not been able to decide if (D) holds for cubic 3-folds, though we suspect it does not hold, at least in general.

While studying (D), often, a related point property (cf. Section 4) is particularly useful (both in disproving and proving (D)). The point property is closely related to the property for a point to be a complete intersection; this property was 
extensively studied in [27]. However, it seems likely that it is a strictly weaker property: we can verify it for a cubic 3 -fold, for example.

(D) makes sense for smooth affine varieties as well. Here, Serre's construction implies that (D) holds for all affine surfaces. We conjecture that there exist smooth affine complex 3-folds for which (D) fails. We formulate a question on projective modules, a negative answer to which gives examples of smooth affine varieties for which (D) would fail (see Section 5).

There are also topological versions of (D). Here, the question comes in several flavours: we may ask if the property holds

(i) for a smooth manifold, with an arbitrary smooth vector bundle of appropriate rank (this property is denoted $\left(D_{r}\right)$ ), or

(ii) for an oriented manifold, and an oriented vector bundle (this is denoted $\left.\left(D_{o}\right)\right)$, or

(iii) (in appropriate cases) for an even dimensional almost complex smooth manifold $X$, and a compatible complex vector bundle on $X \times X$ (this is called property $\left.\left(D_{c}\right)\right)$.

Some of these questions are investigated in this paper, in Section 6.

There are some obvious remarks, analogous to those in the algebraic case: each of the diagonal properties is compatible with products, $\left(D_{r}\right)$ holds for 1-manifolds, and real flag varieties $S L_{n}(\mathbb{R}) / P$, and $\left(D_{c}\right)$ holds for Riemann surfaces, and for flag varieties $S L_{n}(\mathbb{C}) / P$. However, orientable real flag varieties need not satisfy $\left(D_{o}\right)$ (it fails for odd dimensional real projective spaces, as we see in Theorem 3).

We obtain a homotopy theoretic translation of the diagonal property (of any of the above types): if $Y$ is the complement of an open tubular neighborhood of the diagonal in $X \times X$, then the property is equivalent to the extendability of a certain vector bundle on $\partial Y$ to the whole of $Y$; this can be viewed as an extension problem for a map to a classifying space, leading to a possible obstruction theoretic approach (see Lemma 5).

We also have several explicit results, obtained by different methods, which are as follows.

Theorem 3. (1) The sphere $S^{n}$ has $\left(D_{r}\right)$ iff $n=1,2,4$ or 8 . It has $\left(D_{o}\right)$ iff $n=2,4,8$. It has $\left(D_{c}\right)$ iff $n=2$.

(2) A compact oriented odd dimensional manifold $X$ does not have $\left(D_{o}\right)$, and does not have $\left(D_{r}\right)$ if $H^{1}(X, \mathbb{Z} / 2 \mathbb{Z})=0$.

(3) Any compact almost complex 4-manifold has $\left(D_{c}\right)$. If a compact almost complex 6-manifold $X$ satisfying $H^{1}(X, \mathbb{Z})=0$ and $H^{2}(X, \mathbb{Z}) \cong \mathbb{Z}$ has $\left(D_{c}\right)$, then it has a spin structure. 
(4) A (smooth) complex quadric projective hypersurface $X \subset \mathbb{C P}^{2 n}$ does not have $\left(D_{c}\right)$, unless $n=1$.

There are other contexts in which analogues of (D) make sense, that we do not investigate here: e.g. for compact complex manifolds, and for Stein manifolds, or over non-algebraically closed fields. Thus, one may ask if there exist Stein manifolds of dimension $\geq 3$ for which the diagonal is not the zero set, with multiplicity 1 , of a section of a holomorphic vector bundle. Similarly, for a projective smooth surface over a number field, which is geometrically rational, the validity of (D) might provide a subtle obstruction to rationality over the given field.

\section{Preliminaries on (D)}

2.1. Some general remarks. Let $\mathcal{J}_{\Delta} \subset \mathcal{O}_{X \times X}$ denote the ideal sheaf of $\Delta \subset$ $X \times X$. If a variety $X$ satisfies (D), then the cotangent sheaf is isomorphic to the restriction of $\mathcal{E}^{*}$ to the diagonal:

$$
\mathcal{E}_{\mid \Delta}^{*}=\mathcal{J}_{\Delta} / \mathcal{J}_{\Delta}^{2} \cong \Omega_{X}^{1}
$$

(via the isomorphism $\Delta \cong X$ ), so that it is locally free and consequently $X$ is smooth.

Setting $\mathcal{L}=\operatorname{det}\left(\mathcal{E}^{*}\right)$, we have

$$
\mathcal{L}_{\mid \Delta}=\omega_{\Delta} \cong \omega_{X} .
$$

We have also the following expression for the fundamental class of $\Delta$ :

$$
[\Delta]=c_{\operatorname{dim}(X)}(\mathcal{E})
$$

by the Grothendieck formula [14], Théorème 2 .

2.2. (D) for proper smooth varieties. In this section, we assume that $X$ is a proper smooth variety of dimension $n \geq 2$ over an algebraically closed field $k$. We make a preliminary analysis of the condition (D), motivated by Serre's construction of vector bundles of rank 2 (cf. [31]), and some variants.

Consider the exact sequence

$$
0 \rightarrow \mathcal{J}_{\Delta} \rightarrow \mathcal{O}_{X \times X} \rightarrow \mathcal{O}_{\Delta} \rightarrow 0
$$

For any line bundle $\mathcal{L}$ on $X \times X$, if we apply the functor $\operatorname{Hom}(-, \mathcal{L}$ ) (and its derived functors) to the exact sequence (4), we get the following exact sequence of global Ext's (all Ext's without subscripts are taken over $X \times X$ ):

$$
H^{n-1}(X \times X, \mathcal{L}) \rightarrow \operatorname{Ext}^{n-1}\left(\mathcal{J}_{\Delta}, \mathcal{L}\right) \stackrel{\beta}{\rightarrow} \operatorname{Ext}^{n}\left(\mathcal{O}_{\Delta}, \mathcal{L}\right) \stackrel{\alpha}{\rightarrow} H^{n}(X \times X, \mathcal{L}) .
$$

Suppose now that $\mathcal{E}$ is a vector bundle (i.e., a locally free sheaf) of rank $n$ on $X \times X$, with a global section $s: \mathcal{O}_{X \times X} \rightarrow \mathcal{E}$, whose zero scheme is the 
diagonal $\Delta$. We then have the (truncated) Koszul complex associated with the dual $s^{*}: \mathcal{E}^{*} \rightarrow \mathcal{O}_{X \times X}$,

$$
0 \rightarrow \operatorname{det}\left(\mathcal{E}^{*}\right) \rightarrow \cdots \rightarrow \mathcal{E}^{*} \stackrel{s^{*}}{\rightarrow} \mathcal{J}_{\Delta} \rightarrow 0
$$

which determines an element $[s] \in \operatorname{Ext}^{n-1}\left(\mathcal{J}_{\Delta}, \operatorname{det}\left(\mathcal{E}^{*}\right)\right)$. On splicing this with the short exact sequence (4), we obtain the Koszul resolution of $\mathcal{O}_{\Delta}$, and a resulting element

$$
\beta([s]) \in \operatorname{Ext}^{n}\left(\mathcal{O}_{\Delta}, \operatorname{det}\left(\mathcal{E}^{*}\right)\right),
$$

where $\beta$ is the map in (5), with the choice $\mathcal{L}=\operatorname{det}\left(\mathcal{E}^{*}\right)$. This amounts to the assertion that the boundary map $\beta$ may be viewed as a Yoneda product with the class of the extension (4).

Our basic criterion for testing if (D) holds is based on the following result, which is folklore (see the discussion in [8], where this is the condition that the subscheme $\Delta \subset X \times X$ is "strongly subcanonical").

Proposition 1. Let $\mathcal{L}$ be a line bundle on $X \times X$ whose restriction to $\Delta$ coincides with $\omega_{\Delta}$. Assume that there exist a rank $n$ vector bundle $\mathcal{E}$ on $X \times X$ with $\operatorname{det}\left(\mathcal{E}^{*}\right)=\mathcal{L}$, and a section $s \in \Gamma(X \times X, \mathcal{E})$ satisfying $\operatorname{Im}\left(s^{*}\right)=\mathcal{J}_{\Delta}$. Then the map $\alpha$ in the exact sequence (5) vanishes. The converse holds if $n=\operatorname{dim}(X)=2$.

Proof. By the assumption, $\mathcal{L}$, restricted to $\Delta$, coincides with $\omega_{\Delta}$. By Serre duality on $X \times X$,

$$
\operatorname{Ext}^{n}\left(\mathcal{O}_{\Delta}, \mathcal{L}\right)^{*} \cong H^{n}\left(\Delta, \mathcal{L}^{*} \otimes \omega_{X \times X \mid \Delta}\right)=H^{n}\left(\Delta, \omega_{\Delta}\right) .
$$

Hence the space $\operatorname{Ext}^{n}\left(\mathcal{O}_{\Delta}, \mathcal{L}\right)$ is 1-dimensional.

We also have isomorphisms of sheaf Ext's

$$
\underline{\operatorname{Ext}^{i}}\left(\mathcal{O}_{\Delta}, \mathcal{L}\right) \cong\left\{\begin{array}{cl}
0 & \text { if } i \neq n \\
\underline{\operatorname{Hom}}_{\mathcal{O}_{\Delta}}\left(\operatorname{det}\left(\mathcal{J}_{\Delta} / \mathcal{J}_{\Delta}^{2}\right), \mathcal{L}\right. & \text { if } i=n
\end{array}\right.
$$

(cf. [15], III, $\S 7$ ) because $\Delta \subset X \times X$ is a nonsingular subvariety of codimension $n$, with

$$
\underline{\operatorname{Hom}}_{\mathcal{O}_{\Delta}}\left(\operatorname{det}\left(\mathcal{J}_{\Delta} / \mathcal{J}_{\Delta}^{2}\right), \mathcal{L}_{\mid \Delta}\right) \cong \omega_{\Delta}^{-1} \otimes \mathcal{L}_{\mid \Delta} \cong \mathcal{O}_{\Delta} .
$$

This implies that the canonical map

$$
\gamma: \operatorname{Ext}^{n}\left(\mathcal{O}_{\Delta}, \mathcal{L}\right) \rightarrow H^{0}\left(X \times X, \underline{\operatorname{Ext}}^{n}\left(\mathcal{O}_{\Delta}, \mathcal{L}\right)\right) \cong H^{0}\left(\Delta, \mathcal{O}_{\Delta}\right)
$$

is an isomorphism of 1-dimensional vector spaces.

Now, if there exists a vector bundle $\mathcal{E}$ with $\operatorname{det}\left(\mathcal{E}^{*}\right)=\mathcal{L}$, and a section $s \in$ $\Gamma(X \times X, \mathcal{E})$ with zero scheme equal to the diagonal, then there exists $\beta[s] \in$ 
$\operatorname{Ext}^{n}\left(\mathcal{O}_{\Delta}, \mathcal{L}\right)$ which is in the kernel of $\alpha$. But $\beta[s]$ is the class of the Koszul resolution of $\mathcal{O}_{\Delta}$, which is a generator of the 1-dimensional vector space $H^{0}(X \times$ $\left.X, \operatorname{Ext}^{n}\left(\mathcal{O}_{\Delta}, \mathcal{L}\right)\right)$. Hence $\alpha$, which has a non-trivial kernel, and whose domain is 1-dimensional, must be the zero map.

When $n=2$, if $\alpha=0$, the isomorphism $\gamma$ implies the existence of an element $[s] \in \operatorname{Ext}^{1}\left(\mathcal{J}_{\Delta}, \mathcal{L}\right)$ whose image $\beta([s]) \in \operatorname{Ext}^{2}\left(\mathcal{O}_{\Delta}, \mathcal{L}\right)$ generates this 1-dimensional vector space. This element $[s]$ determines an extension, that is to say, a vector bundle $\mathcal{E}$ and section $s$ giving rise to a 3 -term exact sequence (6); this is the Serre construction.

We call a line bundle $\mathcal{M}$ on $X$ cohomologically trivial if $H^{i}(X, \mathcal{M})=0$ for all $i$. If $\mathcal{M}$ is cohomologically trivial on a smooth proper variety $X$, then by Serre duality, so is $\mathcal{M}^{-1} \otimes \omega_{X}$.

We now discuss the criterion which we actually use in studying the property (D), in many instances. This leads to a good understanding of (D) in the surface case, and a nontrivial necessary condition for (D) to hold, in the higher dimensional case.

Theorem 4. (i) Denote by $p_{1}, p_{2}: X \times X \rightarrow X$ the two projections. Suppose that

$$
\operatorname{Pic}(X \times X) \cong p_{1}^{*} \operatorname{Pic}(X) \oplus p_{2}^{*} \operatorname{Pic}(X),
$$

and that $X \times X$ supports a vector bundle $\mathcal{E}$ with a section s such that $(D)$ holds. Then there exists a cohomologically trivial line bundle $\mathcal{M}$ on $X$, such that $\operatorname{det}(\mathcal{E})=$ $p_{1}^{*} \mathcal{M}^{-1} \otimes p_{2}^{*}\left(\mathcal{M} \otimes \omega_{X}^{-1}\right)$.

(ii) If $\operatorname{dim}(X)=2$, and there exists a cohomologically trivial line bundle on $X$, then (D) holds for $X$.

Proof. Suppose that there exists a vector bundle $\mathcal{E}$ on $X \times X$ of rank $n$ such that the diagonal is the zero scheme of its section $s$. Let $\mathcal{L}=\operatorname{det}\left(\mathcal{E}^{*}\right)$, and form the corresponding exact sequence (5). From Proposition 1, we must have $\alpha=0$.

Now consider the dual linear transformation to $\alpha$ :

$$
\alpha^{*}: H^{n}(X \times X, \mathcal{L})^{*} \rightarrow \operatorname{Ext}^{n}\left(\mathcal{O}_{\Delta}, \mathcal{L}\right)^{*} \cong H^{n}\left(\Delta, \omega_{\Delta}\right)=k .
$$

Using (11) and (2), choose $\mathcal{M} \in \operatorname{Pic}(X)$ such that

$$
\mathcal{L}=\operatorname{det}\left(\mathcal{E}^{*}\right) \cong p_{1}^{*}(\mathcal{M}) \otimes p_{2}^{*}\left(\mathcal{M}^{-1} \otimes \omega_{X}\right) .
$$


By Serre duality on $X \times X$, we get that

$H^{n}(X \times X, \mathcal{L})^{*} \cong H^{n}\left(X \times X, \mathcal{L}^{-1} \otimes \omega_{X \times X}\right) \cong H^{n}\left(X \times X, p_{1}^{*}\left(\mathcal{M}^{-1} \otimes \omega_{X}\right) \otimes p_{2}^{*} \mathcal{M}\right)$.

From the Künneth formula, we have

(13) $H^{n}\left(X \times X, p_{1}^{*}\left(\mathcal{M}^{-1} \otimes \omega_{X}\right) \otimes p_{2}^{*}(\mathcal{M})\right)=\oplus_{i=0}^{n} H^{i}\left(X, \mathcal{M}^{-1} \otimes \omega_{X}\right) \otimes H^{n-i}(X, \mathcal{M})$.

Further, on any summand on the right, the induced map

$H^{i}\left(X, \mathcal{M}^{-1} \otimes \omega_{X}\right) \otimes H^{n-i}(X, \mathcal{M}) \hookrightarrow H^{n}\left(X \times X, p_{1}^{*}\left(\mathcal{M}^{-1} \otimes \omega_{X}\right) \otimes p_{2}^{*}(\mathcal{M})\right) \stackrel{\alpha^{*}}{\rightarrow} H^{n}\left(\Delta, \omega_{\Delta}\right)=k$

coincides with the Serre duality pairing on cohomology of $X$, and is hence a non-degenerate bilinear form, for each $0 \leq i \leq n$.

Thus, the dual map to $\alpha$ vanishes if and only if all of the summands on the right side of (13) vanish, which amounts to saying that $\mathcal{M}$ is cohomologically trivial.

Conversely, if $\mathcal{M}$ is cohomologically trivial, then in the exact sequence (5) determined by the line bundle

$$
\mathcal{L}=p_{1}^{*} \mathcal{M} \otimes p_{2}^{*}\left(\mathcal{M}^{-1} \otimes \omega_{X}\right),
$$

the map $\alpha$ is the zero map, by reversing the above argument. Hence, if $n=2$, we deduce that $X \times X$ supports a vector bundle $\mathcal{E}$ of rank 2 and a section $s$ with zero scheme $\Delta$, by the surface case of Proposition 1 .

In particular, if the isomorphism (11) holds for $X$, and $X$ supports no cohomologically trivial bundle, then $X$ fails to have (D).

In order to apply Theorem 4 in various situations, we need to be able to verify the hypothesis (11) on the Picard group. This property is well understood, and the facts are recapitulated below.

Lemma 1. Let $X$ be any smooth proper variety over an algebraically closed field $k$.

(i) The isomorphism (11) holds if and only if $\operatorname{Pic}(X)$ is a finitely generated abelian group.

(ii) If $H^{1}\left(X, \mathcal{O}_{X}\right)=0$, then (11) holds.

Proof. (i) Let $\underline{\operatorname{Pic}}^{0}(X)$ denote the neutral component (identity component) of the Picard scheme of $X$, in the sense of Grothendieck. Then $\underline{\operatorname{Pic}}^{0}(X)$ is a projective connected $k$-group scheme, such that 
(a) its Lie algebra is naturally identified with the vector space $H^{1}\left(X, \mathcal{O}_{X}\right)$;

(b) the associated reduced group scheme is an abelian variety, the classical Picard variety (in the sense of Weil).

The group of connected components of the Picard scheme is identified with the Néron-Severi group, which is always a finitely generated abelian group. The group of $k$-rational points of a positive dimensional abelian variety is not finitely generated. Thus, $\operatorname{Pic}(X)$ is a finitely generated group precisely when $\underline{\operatorname{Pic}}^{0}(X)$ is a 0 -dimensional, local $k$-group scheme (in characteristic 0 , this means it is 0 ). The connected group scheme $\underline{\operatorname{Pic}}^{0}(X)$ is of course 0 if its Lie algebra $H^{1}\left(X, \mathcal{O}_{X}\right)$ vanishes.

In any case, if $\mathcal{L}$ is any line bundle on $X \times X$, which (for some fixed base point $\left.x_{0} \in X\right)$ is trivialized on $X \times\left\{x_{0}\right\}$ as well as on $\left\{x_{0}\right\} \times X$, then by the universal property of the Picard scheme, $\mathcal{L}$ determines a morphism of $k$-schemes $f_{\mathcal{L}}: X \rightarrow \underline{\operatorname{Pic}}(X)$, which maps $x_{0}$ to the identity point, so that $\mathcal{L}$ is the pull-back under $1_{X} \times f_{\mathcal{L}}: X \times X \rightarrow X \times \underline{\operatorname{Pic}}(X)$ of a suitable Poincaré bundle. Clearly $f_{\mathcal{L}}$ factors through the neutral component, and then through the corresponding reduced scheme, which is the identity point when $\operatorname{Pic}(X)$ is finitely generated. Hence $\mathcal{L}$ is the trivial bundle. This clearly implies (11).

On the other hand, suppose $\operatorname{Pic}(X)$ is not finitely generated, which is to say that $\underline{\operatorname{Pic}}^{0}(X)_{\text {red }}$ is a positive dimensional abelian variety. We claim that (11) does not hold in this case.

Indeed, the dual abelian variety to $\underline{\operatorname{Pic}}^{0}(X)_{\text {red }}$ is the Albanese variety $\operatorname{Alb}(X)$, in the sense of Weil, which is an abelian variety of the same dimension $(>0)$ as the Picard variety; further, there is a morphism $f: X \rightarrow \operatorname{Alb}(X)$ such that $f\left(x_{0}\right)=0$, which is universal among morphisms of pairs $\left(X, x_{0}\right) \rightarrow(A, 0)$ with $A$ any abelian variety. As a consequence, the $k$-points of the image variety $f(X)$ generate $\operatorname{Alb}(X)$ as a group, and, in particular, $f$ has positive dimensional image.

From the duality theory of abelian varieties, we know that the Albanese and Picard varietes of $X$, being mutually dual, are also isogenous. This means that, composing $f$ with an isogeny, we can construct a morphism $g: X \rightarrow \underline{\operatorname{Pic}}^{0}(X)_{\text {red }}$ with $g\left(x_{0}\right)$ equal to the neutral element, whose image is a positive dimensional subvariety, whose $k$-points generate the group of points of the abelian variety 
$\underline{\operatorname{Pic}}^{0}(X)_{\text {red. }}$ The pullback of the Poincaré line bundle under the morphism

$$
1_{X} \times g: X \times X \rightarrow X \times \underline{\operatorname{Pic}}^{0}(X)
$$

is a line bundle on $X \times X$ which is non-trivial, but is trivial when restricted to $X \times\left\{x_{0}\right\}$ or to $\left\{x_{0}\right\} \times X$. Hence (11) fails.

(ii) This follows from "Cohomology and Base Change" (cf., e.g., [15, Theorem 12.11] and comment on p. 292).

\section{MAin RESUlts FOR SURFACES}

We now explore (D) for surfaces. Our main references on the theory of surfaces are: [2], [3], [13], and [15]. From Theorem 4, for a surface $X,(\mathrm{D})$ is more or less equivalent to the existence of a cohomologically trivial line bundle on $X$.

Before a more systematic discussion, we consider the following simple result, since it was our first example of failure of (D).

Proposition 2. Let $X$ be a generic complex algebraic K3 surface. Then (D) fails for $X$.

Proof. It follows, e.g., from [13], p. 594, that $\operatorname{Pic}(X)=\mathbb{Z} \cdot \mathcal{O}_{X}(1)$, where $\mathcal{O}_{X}(1)$ is an ample line bundle on $X$. Let $\mathcal{O}_{X}(1) \cong \mathcal{O}_{X}(D)$ for a divisor $D$; then $d:=$ $D^{2}>0$.

Since $X$ has a trivial canonical bundle and $\chi\left(X, \mathcal{O}_{X}\right)=2$, the Riemann-Roch theorem implies that for any integer $n$,

$$
\chi\left(X, \mathcal{O}_{X}(n)\right)=\frac{n^{2} d}{2}+2 \geq 2 .
$$

Consequently, since every line bundle $\mathcal{M}$ is isomorphic to some $\mathcal{O}_{X}(n), \mathcal{M}$ must have some nontrivial cohomology. The assertion now follows from Theorem 4(i) (and Lemma 1).

We now proceed more systematically.

3.1. (D) within a birational class. A classical result in surface theory asserts that any smooth proper (equivalently, projective) algebraic surface is obtained from a (relative) minimal model by a succession of blow ups of points (cf., e.g., [15] III, Theorem 5.8 and II, 4.10.2). Thus, the following result reduces (D) for 
surfaces to the question of finding cohomologically trivial line bundles on relative minimal models.

Proposition 3. Given a birational proper morphism $f: X \rightarrow Y$ of smooth surfaces, if $Y$ supports a cohomologically trivial line bundle $\mathcal{M}$, then the pullback $f^{*} \mathcal{M}$ is also cohomologically trivial.

Proof. The morphism $f$ is a composition of point blow ups, as noted above. By induction on the number of blow ups, we thus reduce to the case when $f$ is the blow up of 1 point. In this case, it is standard that $f_{*} \mathcal{O}_{X}=\mathcal{O}_{Y}$ and $R^{i} f_{*} \mathcal{O}_{X}=0$ for all $i>0$ (see [15], V, 3.4, for example). From the projection formula ([15], III, Ex. 8.3, for example), it follows that for any cohomologically trivial line bundle $\mathcal{M}$ on $Y$, the pullback $f^{*} \mathcal{M}$ is cohomologically trivial on $X$.

Corollary 1. If a smooth projective surface $X$ admits a morphism to a relative minimal model $Y$ which supports a cohomologically trivial line bundle, then $X$ has (D).

Let us note also:

Corollary 2. If $f: X \rightarrow Y$ is a birational morphism of smooth projective surfaces with finitely generated Picard groups, and $Y$ has (D), then $X$ has (D).

On the other hand, we claim that any "sufficiently non-minimal" surface has a cohomologically trivial line bundle, and hence satisfies (D).

Theorem 5. If $X$ is any smooth projective surface over an algebraically closed field $k$, there is a birational proper morphism $f: Y \rightarrow X$ of smooth projective surfaces such that $Y$ satisfies $(D)$.

Proof. Choose a line bundle $\mathcal{L}$ on $X$ so that $H^{i}(X, \mathcal{L})=0$ for $i>0$ (by Serre vanishing, $H^{i}\left(X, \mathcal{O}_{X}(n)\right)=0$ for all $i>0$, and any $n>>0$, so such an $\mathcal{L}$ certainly exists). If $H^{0}(X, \mathcal{L})=0$, then $\mathcal{L}$ is cohomologically trivial, hence (D) holds for $X$ itself. So let us assume that $\operatorname{dim} H^{0}(X, \mathcal{L})=r>0$.

For any $x \in X$, let $\mathcal{L}(x)=\mathcal{L}_{x} \otimes_{\mathcal{O}_{X, x}} k(x)$ denote the fiber of $\mathcal{L}$ at $x$; this is a 1-dimensional vector space over $k=k(x)$.

We now state a standard lemma, whose proof is left to the reader. 
Lemma 2. Let $X$ be a projective variety over an algebraically closed field, and $\mathcal{L} \in \operatorname{Pic}(X)$ with $\operatorname{dim} H^{0}(X, \mathcal{L})=r>0$. Then for any $r$ general points $x_{1}, \ldots, x_{r}$ of $X$, the induced map of $r$-dimensional vector spaces

$$
f_{r}: H^{0}(X, \mathcal{L}) \rightarrow \oplus_{i=1}^{r} \mathcal{L}\left(x_{i}\right)
$$

is an isomorphism. ${ }^{2}$

Now, let $\mathcal{L}$ be as before on our surface $X$, and let $x_{1}, \ldots, x_{r}$ be chosen as in the lemma. Let $f: Y \rightarrow X$ be the blow up of the points $x_{1}, \ldots, x_{r}$, and let $E_{1}, \ldots, E_{r}$ be the corresponding exceptional curves. Consider the line bundle

$$
\mathcal{M}=f^{*} \mathcal{L} \otimes \mathcal{O}_{Y}\left(-E_{1}-E_{2}-\cdots-E_{r}\right)
$$

on $Y$. We claim that $\mathcal{M} \in \operatorname{Pic}(Y)$ is cohomologically trivial, and hence $Y$ satisfies (D). Indeed, it is easy to see that if $S=\left\{x_{1}, \ldots, x_{r}\right\}$, and $\mathcal{J}_{S}$ is the ideal sheaf of $S$, then

$$
R^{i} f_{*} \mathcal{M}=\mathcal{L} \otimes R^{i} f_{*} \mathcal{O}_{Y}\left(-E_{1}-\cdots-E_{r}\right)=0
$$

if $i>0$, and $f_{*} \mathcal{M}=\mathcal{L} \otimes \mathcal{J}_{S}$. Thus, from the Leray spectral sequence for $f$, it follows at once that

$$
H^{i}(Y, \mathcal{M})=H^{i}\left(X, \mathcal{L} \otimes \mathcal{J}_{S}\right) .
$$

There is an exact sequence of sheaves

$$
0 \rightarrow \mathcal{L} \otimes \mathcal{J}_{S} \rightarrow \mathcal{L} \rightarrow \mathcal{L} \otimes \mathcal{O}_{S} \rightarrow 0,
$$

where we may identify

$$
\mathcal{L} \otimes \mathcal{O}_{S}=\oplus_{i=1}^{r} \mathcal{L}\left(x_{i}\right)_{x_{i}}
$$

(here, for a point $x$ and abelian group $A$, we let $A_{x}$ denote the skyscraper sheaf with stalk $A$ at $x)$. Then clearly $H^{i}\left(X, \mathcal{L} \otimes \mathcal{O}_{S}\right)=0$ for $i>0$, while

$$
H^{0}(X, \mathcal{L}) \rightarrow H^{0}\left(X, \mathcal{L} \otimes \mathcal{O}_{S}\right)=\oplus_{i=1}^{r} \mathcal{L}\left(x_{i}\right)
$$

is the map $f_{r}$ considered above, which (by the choice of the set $S$ ) is an isomorphism.

Hence, the long exact cohomology sequence for the above sequence of sheaves implies that $\mathcal{L} \otimes \mathcal{J}_{S}$ is cohomologically trivial on $X$, and so $\mathcal{M}$ is cohomologically trivial on $Y$.

\footnotetext{
${ }^{2}$ This says that, if we blow up the base scheme to get a morphism to projective space $\mathbb{P}^{r-1}$, the linear span of the image is the whole projective space, so that there exist $r$ linearly independent points in the image.
} 
3.2. Ruled surfaces. We shall examine now the case of birationally ruled surfaces.

It is useful for us to make explicit the following observation (which is of course well-known).

Lemma 3. Any smooth projective curve $Y$ over an algebraically closed field supports a cohomologically trivial line bundle $\mathcal{L}$.

Proof. This is clear if $Y=\mathbb{P}^{1}$ (take $\mathcal{L}=\mathcal{O}_{Y}(-1)$ ). If $Y$ has genus $g>0$, the isomorphism classes of line bundles of degree $g-1$ on $Y$ are parametrized by a $g$-dimensional variety $J^{g-1}(Y)$ (it is a principal homogeneous space under the Jacobian variety $J(Y)$ ). On the other hand, the subvariety parametrizing line bundles with a non-zero section is the image of the natural morphism $S^{g-1}(Y) \rightarrow$ $J^{g-1}(Y)$, with domain the $(g-1)$ st symmetric power of $Y$, which is the parameter variety for effective divisors of degree $g-1$. Clearly, this is not surjective; a point in the complement of the image corresponds to a line bundle $\mathcal{L}$ of degree $g-1$ on $Y$ such that $\mathcal{L}$ has no non-zero global sections. Now, the Riemann-Roch theorem on $Y$ implies $\mathcal{L}$ has vanishing Euler characteristic, hence is cohomologically trivial.

Proposition 4. Let $X$ be a birationally ruled surface. Then $X$ admits a cohomologically trivial line bundle, and, in particular, (D) holds for $X$.

Proof. We may as well assume $X ¥ \mathbb{P}^{2}$, since $\mathcal{O}_{\mathbb{P}^{2}}(-1)$ is cohomologically trivial. Then, from the classification of birationally ruled surfaces, we know that $X$ is birational to $Y \times \mathbb{P}^{1}$ for some smooth projective curve $Y$, such that there is a morphism $\pi: X \rightarrow Y$ with general fiber $\mathbb{P}^{1}$. The morphism $\pi$ has a factorization as a composition

$$
X \rightarrow \bar{X} \rightarrow Y
$$

where $X \rightarrow \bar{X}$ is a composition of point blow ups, and $\bar{X} \rightarrow Y$ is a $\mathbb{P}^{1}$-bundle. By Proposition 3 , it suffices to show $\bar{X}$ supports a cohomologically trivial line bundle.

Hence we may, without loss of generality, assume that $\pi: X \rightarrow Y$ is a $\mathbb{P}^{1}$ bundle. We now note that if $\mathcal{L}$ is cohomologically trivial on $Y$ (and such a line bundle exists, by Lemma 3 ), then $\pi^{*} \mathcal{L}$ is cohomologically trivial on the surface $X$. This follows from the Leray spectral sequence, because for the $\mathbb{P}^{1}$-bundle $\pi: X \rightarrow Y$, we have $R^{i} \pi_{*} \mathcal{O}_{X}=0$ for $i>0$, and $\pi_{*} \mathcal{O}_{X}=\mathcal{O}_{Y}$. 


\subsection{Surfaces of Kodaira dimension $\mathbf{0 .}$}

Proposition 5. An abelian surface supports a cohomologically trivial line bundle. Thus (D) holds for abelian surfaces.

Proof. It is well known (cf., e.g., [24], Sect. 8) that for an abelian variety $X$, any line bundle $\mathcal{M}$ on $X$ which is non-trivial, but is algebraically equivalent to 0 (i.e., having a nontrivial class in $\operatorname{Pic}^{0}(X)$ ), is cohomologically trivial.

Note that in the surface case, after showing that $H^{0}(X, \mathcal{M})=0$ (loc.cit.), one can argue as follows. Since $\omega_{X}$ is trivial, we get by Serre duality that $H^{2}\left(X, \mathcal{M}^{-1}\right)=0$. But, exchanging the roles of $\mathcal{M}$ and $\mathcal{M}^{-1}$, this implies that $H^{2}(X, \mathcal{N})=0$. On the other hand, we have by the Riemann-Roch theorem that $\chi(\mathcal{M})=0$, so that we also have $H^{1}(X, \mathcal{M})=0$. Applying Theorem 4(ii), the assertion follows.

Proposition 6. A K3 surface $X$ with two disjoint smooth rational curves supports a cohomologically trivial line bundle, and (D) holds for it.

Proof. Let $D_{1}$ and $D_{2}$ be two disjoint smooth rational curves on $X$. Let

$$
\mathcal{M}=\mathcal{O}\left(D_{1}-D_{2}\right) \text {. }
$$

We claim that $\mathcal{M}$ is cohomologically trivial. By the adjunction formula, the curves $D_{1}, D_{2}$ are $(-2)$ curves. This implies that $H^{0}(X, \mathcal{M})=0$. By Serre duality, invoking that $\omega_{X}$ is trivial, we get that $H^{2}\left(X, \mathcal{M}^{-1}\right)=0$. But, exchanging the roles of $D_{1}$ and $D_{2}$, this gives $H^{2}(X, \mathcal{M})=0$. The Riemann-Roch theorem for a K3 surface reads (with $\mathcal{L}=\mathcal{O}(D)$ ):

$$
\chi(\mathcal{L})=\frac{1}{2} D^{2}+2 .
$$

Hence we get the vanishing $\chi(\mathcal{M})=0$, and consequently we have $H^{1}(X, \mathcal{M})=0$. Applying Theorem 4(ii), the assertion follows.

Corollary 3. For the Kummer surface, (D) holds.

In the rest of this section, we assume the ground field is $\mathbb{C}$, for simplicity.

We use the term hyperelliptic surface to mean a complex surface $X$ which is a quotient of a product $E \times C$ by the diagonal action of a finite abelian group $G$ which acts on each of the factors, where $E$ is an elliptic curve on which $G$ acts 
faithfully by translations, while $C$ is a smooth projective curve of genus $>0$ such that $C / G \cong \mathbb{P}^{1}$. The surface

$$
X=(E \times C) / G
$$

admits a morphism to $C / G \cong \mathbb{P}^{1}$ with fibers which are elliptic curves. Such a surface has Kodaira dimension 0 if $C$ is also elliptic; else, it has Kodaira dimension 1.

Proposition 7. A hyperelliptic surface (as above) admits a cohomologically trivial line bundle, and (D) holds for it.

Proof. First observe that since $q: E \times C \rightarrow X$ is a finite covering of complex surfaces, the cohomology of any line bundle on $X$ injects into the cohomology of its pull-back to $E \times C$. Hence, if we show that there is some line bundle $\mathcal{M}$ on $X$ whose pull-back to $E \times C$ is cohomologically trivial, then $\mathcal{M}$ itself is cohomologically trivial.

Since $G$ is a finite group of translations on $E$, the map $\pi: E \rightarrow E / G$ is an isogeny of elliptic curves. We can find a nontrivial line bundle $\mathcal{L}$ on $E / G$ of degree 0 , whose pull-back $\pi$ is a non-trivial line bundle on $E$, also of degree 0 (i.e., $\mathcal{L}$ considered as a point of $E / G \cong \operatorname{Pic}^{0}(E / G)$ is not in the kernel of the dual isogeny to $\pi$ ).

In particular, $\pi^{*} \mathcal{L}$ is cohomologically trivial on $E$. If $p_{1}: E \times C \rightarrow E$ is the projection, the pullback $p_{1}^{*} \pi^{*} \mathcal{L}$ on $E \times C$ is again cohomologically trivial, from the Künneth formula.

There is a commutative diagram, whose horizontal arrows are quotients mod $G$, with induced vertical arrow $f$

$$
\begin{array}{cc}
E \times C \stackrel{q}{\rightarrow} \quad X \\
p_{1} \downarrow & \downarrow f \\
E & \stackrel{\pi}{\rightarrow} E / G
\end{array}
$$

Thus $\mathcal{M}=f^{*} \mathcal{L}$ has the property that $q^{*} \mathcal{M}=p_{1}^{*} \pi^{*} \mathcal{L}$ is cohomologically trivial; hence $\mathcal{M}$ is cohomologically trivial on $X$.

We shall now discuss the case of Enriques surfaces (surfaces with $p_{a}=p_{g}=0$ whose canonical line bundle has order 2).

Proposition 8. Any complex Enriques surface supports a cohomologically trivial line bundle, and hence has (D). 
Proof. First, suppose we have a smooth $(-2)$ curve $E$ on the Enriques surface $X$ (i.e., $E \cong \mathbb{P}^{1}$, and $E^{2}=-2$ ). We then claim $\mathcal{O}_{X}(-E)$ is cohomologically trivial (and so the diagonal property holds). Indeed, we have $\chi\left(X, \mathcal{O}_{X}(-E)\right)=0$ by the Riemann-Roch theorem. Since $\mathcal{O}_{X}(-E)$ is the ideal sheaf of a smooth rational curve, and $X$ has $H^{1}\left(X, \mathcal{O}_{X}\right)=0$, we have

$$
H^{0}\left(X, \mathcal{O}_{X}(-E)\right)=H^{1}\left(X, \mathcal{O}_{X}(-E)\right)=0 .
$$

Hence this ideal sheaf is a cohomologically trivial line bundle.

Now we shall use [2], VIII, Lemmas 16.4, 17.1, 17.2, and 17.3. These results imply that there always exist "half pencils" on an Enriques surface $X$, that is, effective divisors $D$ such that the normal bundle $\mathcal{O}_{D}(D)$ is a line bundle of order 2 , where $D$ is a non-multiple divisor which is an "elliptic configuration", i.e. a member of the Kodaira list in Table 3 on p. 150 in [2], V, Sect. 7. The discussion of case (c) in the same section (cf. top of p. 151) says, in fact, that $D$ is reduced, and is either a smooth elliptic curve, an irreducible curve with one ordinary double point, or a polygon of $b$ curves, each of which is a smooth rational $(-2)$ curve (Kodaira's types $I_{0}, I_{1}$ and $I_{b}$, respectively).

If we have a half pencil of type $I_{b}$ with $b \geq 2$, we have a smooth $(-2)$ curve $E$ on the surface. Then as seen above, $\mathcal{O}_{X}(-E)$ is cohomologically trivial.

The same argument also takes care of the case of "special Enriques surfaces", on any of which there is a smooth $(-2)$ curve.

So we may assume our Enriques surface is "non-special", with two distinct half pencils $D_{1}, D_{2}$, each of which is irreducible, and is either an elliptic curve, or a singular rational curve with one ordinary double point; further, we may assume their intersection number $\left(D_{1} \cdot D_{2}\right)$ is 1 (cf. [2], VIII, Theorem 17.7). Thus $D_{1}$ and $D_{2}$ must intersect transversally at a point, say $x \in X$, which is a smooth point of each curve.

In this situation, we claim that $\mathcal{O}_{X}\left(D_{1}-D_{2}\right)$ is a cohomologically trivial line bundle.

First, note that $E=D_{1}-D_{2}$ has self-intersection -2 , since $D_{1}^{2}=D_{2}^{2}=0$, while $\left(D_{1} \cdot D_{2}\right)=1$. Since the canonical bundle on an Enriques surface is numerically trivial (it is 2-torsion), and $\chi\left(X, \mathcal{O}_{X}\right)=1$, the Riemann-Roch theorem gives that $\chi\left(X, \mathcal{O}_{X}(E)\right)=0$. 
Next, consider the exact sequence of sheaves

$$
0 \rightarrow \mathcal{O}_{X}\left(D_{1}-D_{2}\right) \rightarrow \mathcal{O}_{X}\left(D_{1}\right) \rightarrow \mathcal{O}_{D_{2}}(x) \rightarrow 0,
$$

where we note that the restriction of $\mathcal{O}_{X}\left(D_{1}\right)$ to the curve $D_{2}$ is the line bundle corresponding to the intersection point $x$. Since $D_{2}$ is an irreducible curve of arithmetic genus $1, \mathcal{O}_{D_{2}}(x)$ has a 1-dimensional space of sections, and vanishing higher cohomology. Hence

$$
H^{0}\left(X, \mathcal{O}_{X}\left(D_{1}\right)\right) \rightarrow H^{0}\left(X, \mathcal{O}_{D_{2}}(x)\right),
$$

being a nonzero map between 1-dimensional vector spaces, is an isomorphism. This means that $\mathcal{O}_{X}\left(D_{1}-D_{2}\right)$ has no nontrivial global sections, and

$$
\mathcal{O}_{X}\left(D_{1}-D_{2}\right) \rightarrow \mathcal{O}_{X}\left(D_{1}\right)
$$

is an isomorphism on higher cohomology.

Now consider the sequence

$$
0 \rightarrow \mathcal{O}_{X} \rightarrow \mathcal{O}_{X}\left(D_{1}\right) \rightarrow \mathcal{O}_{D_{1}}\left(D_{1}\right) \rightarrow 0
$$

where $\mathcal{O}_{D_{1}}\left(D_{1}\right)$, the normal bundle to $D_{1}$, is a line bundle of order 2 . In particular, it is a nontrivial line bundle of degree zero on a curve of arithmetic genus 1 , and thus has no cohomology (it has no sections, and by the Riemann-Roch theorem, its Euler characteristic is equal to zero). Hence $\mathcal{O}_{X} \rightarrow \mathcal{O}_{X}\left(D_{1}\right)$ induces isomorphisms on all cohomology groups. In particular, $\mathcal{O}_{X}\left(D_{1}\right)$ has vanishing cohomology $H^{1}$ and $H^{2}$. Hence so does $\mathcal{O}_{X}\left(D_{1}-D_{2}\right)$.

3.4. Elliptic surfaces. We now pass to elliptic surfaces. Here, we have a result for elliptic surfaces with a section (i.e., for Jacobian fibrations).

Proposition 9. If $f: X \rightarrow C$ is an elliptic fibration over a smooth projective curve $C$, such that $f$ has a section, then $X$ supports a cohomologically trivial line bundle, and so (D) holds.

Proof. Let $D$ be a curve in $X$ mapping isomorphically to $C$ under $f$.

We may replace $f$ by a suitable relatively minimal model (the pull-back of a cohomologically trivial line bundle from the minimal model is of course cohomologicaly trivial). Since $f$ is an elliptic fibration, all fibres of $f$ are connected curves of arithmetic genus 1 . Hence, for any fiber $F, H^{1}\left(F, \mathcal{O}_{F}(D)\right)=0$, while $H^{0}\left(F, \mathcal{O}_{F}(D)\right)$ is 1-dimensional for general fibers $F$ (note that the general fiber is smooth). 
It follows that $R^{1} f_{*} \mathcal{O}_{X}(D)=0$ (see [15] III Ex. 11.8, for example). Hence, for any line bundle $\mathcal{L}$ on $C$, the projection formula identifies

$$
R^{i} f_{*} f^{*} \mathcal{L} \otimes_{\mathcal{O}_{X}} \mathcal{O}_{X}(D)
$$

with

$$
\mathcal{L} \otimes_{\mathcal{O}_{C}} R^{i} f_{*} \mathcal{O}_{X}(D)
$$

Therefore, we obtain an isomorphism

$$
H^{i}\left(C, \mathcal{L} \otimes f_{*} \mathcal{O}_{X}(D)\right) \cong H^{i}\left(X, f^{*} \mathcal{L} \otimes \mathcal{O}_{X}(D)\right)
$$

for any $i \geq 0$. The sheaf $f_{*} \mathcal{O}_{X}(D)$ on $C$ is torsion-free, hence it is a vector bundle. By looking at the generic fiber, we see that it is, in fact, a line bundle.

We now claim that, for any line bundle $\mathcal{M}$ on $C$, there exists a line bundle $\mathcal{L}$ on $C$ so that $\mathcal{L} \otimes \mathcal{M}$ on $C$ is cohomologically trivial. Indeed, by Lemma $3, C$ supports a cohomologically trivial line bundle $\mathcal{L}_{0}$. Choose $\mathcal{L}=\mathcal{L}_{0} \otimes \mathcal{M}^{-1}$.

Remark 1. Proposition 7 for $X=(E \times C) / G$, with $C$ of genus $\geq 2$, also gives examples of complex elliptic surfaces for which (D) holds; some of these surfaces have elliptic fibrations without a section.

3.5. Some surfaces for which (D) fails. We shall give now more surfaces for which (D) fails, because the surface does not support a cohomologically trivial line bundle.

The result below applies to any surface $X$, which is a sufficiently general complete intersection in projective space (or in a homogeneous space $G / P$, where $G$ is a semisimple group, and $P$ a maximal parabolic sugroup), of large enough multi-degree that the canonical bundle of the surface has non-zero sections (this amounts to saying that either $X$ is a K3 surface, or $X$ is of general type). Indeed, since the surface $X$ is a very general member in the corresponding family of surfaces, we have $\operatorname{Pic}(X) \cong \mathbb{Z}$ by the Noether-Lefschetz theorem.

Proposition 10. Suppose $X \supsetneqq \mathbb{P}^{2}$ is a smooth projective surface, with $\operatorname{Pic}(X)=$ $\mathbb{Z}$, such that the ample generator of $\operatorname{Pic}(X)$ has a non-zero section. Then $X$ does not have (D).

Proof. Note first that (11) holds by Lemma 1. So we must show that $X$ does not support any cohomologically trivial line bundle, that is, every power of the ample generator has some non-zero cohomology. 
If $\mathcal{L}$ is a nonnegative power of the ample generator, then clearly $H^{0}(X, \mathcal{L}) \neq 0$, by our assumptions. Suppose now that $\mathcal{L}$ is a negative power of the ample generator. Observe that $\omega_{X}$ is a nonnegative power of the ample generator because (by classification) $X$ is not Fano, since the only Fano surface with Picard group $\mathbb{Z}$ is $\mathbb{P}^{2}$. We conclude that $\mathcal{L}^{-1} \otimes \omega_{X}$ is a strictly positive power of the ample generator, and so, by Serre duality,

$$
H^{2}(X, \mathcal{L})=H^{0}\left(X, \mathcal{L}^{-1} \otimes \omega_{X}\right) \neq 0
$$

The proposition has been proved.

Remark 2. We comment on the hypothesis in Proposition 10 that the ample generator of $\operatorname{Pic}(X)$ has a non-zero section. Any surface $X \neq \mathbb{P}^{2}$ with $\operatorname{Pic}(X)=\mathbb{Z}$ must have trivial canonical bundle, or be of general type, with ample canonical bundle $\omega_{X} \cong \mathcal{O}_{X}(n)$ for some $n>0$, where $\mathcal{O}_{X}(1)$ denotes the ample generator of $\operatorname{Pic}(X)$.

We claim that the canonical bundle of such a complex surface always has a non-zero section, i.e. $p_{g}(X)>0$.

Indeed, suppose that the canonical bundle is ample, with no sections. It follows from Hodge theory that the second Betti number of $X$ equals its Picard number. Hence the topological Euler characteristic of $X$ is 3, while its holomorphic Euler characteristic is 1 . This forces $K_{X}^{2}=9$ by the Noether formula, that is, $X$ is a Fake $\mathbb{P}^{2}$ in the sense of Mumford [25], who first constructed such a surface of general type, using $p$-adic uniformization.

All complex Fake $\mathbb{P}^{2}$ 's have been classified in recent work of Gopal Prasad and Sai-Kee Yeung [30]. From their work (see Theorem 10.1), every Fake $\mathbb{P}^{2}$ has a non-zero torsion subgroup of $\operatorname{Pic}(X)$, and hence does not have Picard group $\mathbb{Z}$.

It is interesting to ask if a complex Fake $\mathbb{P}^{2}$ has (D). Poincaré duality, combined with the Riemann-Roch theorem, gives us that any ample generator of the NéronSeveri group, modulo torsion, has self-intersection 1, and thus vanishing Euler characteristic. Hence (D) holds for $X$ precisely when a line bundle can be found on $X$ which gives an ample generator for $N S(X) /($ torsion), and has vanishing $H^{1}$. 
Remark 3. We do not know an example of a surface $X$ with $\operatorname{Pic}(X)=\mathbb{Z}$, and an ample canonical bundle with a non-zero section, but with $H^{0}\left(X, \mathcal{O}_{X}(1)\right)=0$ for the ample generator $\mathcal{O}_{X}(1)$ of $\operatorname{Pic}(X)$.

\section{Higher Dimensional VARIETies}

4.1. Varieties with Picard group $\mathbb{Z}$. We first consider varieties of dimension $d \geq 3$ with Picard group $\mathbb{Z}$. From the Grothendieck-Lefschetz theorem (cf., e.g., $[16])$, we have $\operatorname{Pic}(X)=\mathbb{Z}$ for any smooth complete intersection $X$, or for a smooth complete intersection of divisors in a homogeneous space $G / P$ with $G$ semisimple, $P$ maximal parabolic (so that $\operatorname{Pic}(G / P)=\mathbb{Z}$ ).

Proposition 11. Let $X$ be a smooth projective variety of dimension $d \geq 3$ over a field. Suppose that

(i) (D) holds for $X$;

(ii) $\operatorname{Pic}(X)=\mathbb{Z}$, and the ample generator of $\operatorname{Pic}(X)$ has a nonzero section.

Then $X$ is a Fano variety, with canonical line bundle $\omega_{X} \cong \mathcal{O}_{X}(-n)$ for some $n \geq 2$.

Proof. Since $X$ has Picard group $\mathbb{Z}$, and (D) holds, Theorem 4 and Lemma 1 imply that $X$ supports a cohomologically trivial line bundle, which must be $\mathcal{O}_{X}(m)$ for some integer $m$. If $\omega_{X}=\mathcal{O}_{X}(r)$, then, by Serre duality, $\mathcal{O}_{X}(m)$ and $\mathcal{O}_{X}(r-m)$ both have $H^{0}=0$. Since the ample generator of $\operatorname{Pic}(X)$ has a nonzero section, we must have $m<0, r-m<0$. Hence $r=(r-m)+m \leq-2$.

Corollary 4. Let $X \subset \mathbb{P}^{n}$ be a smooth complete intersection of multidegree $\left(d_{1}, \ldots, d_{r}\right)$ with $r \leq n-3$, and $\sum_{i} d_{i} \geq n$. Then $X$ does not have $(D)$.

Proof. Such a complete intersection has dimension $n-r \geq 3$, so has Picard group $\mathbb{Z}$ by the Grothendieck-Lefschetz theorem. The canonical bundle of $X$ is

$$
\mathcal{O}_{X}\left(\sum_{i} d_{i}-n-1\right)
$$

where $\sum_{i} d_{i}-n-1 \geq-1$. Hence (D) does not hold for $X$.

Thus, for smooth hypersurfaces in $\mathbb{P}^{4}$, the only cases we need to consider are quadrics and cubics; the case of quadrics is settled below ((D) does not hold, cf. Proposition 12).

For Fano varieties, we similarly have: 
Corollary 5. Let $X$ be a smooth projective complex Fano variety of dimension $\geq 3$ with second Betti number $b_{2}(X)=1$ and $\omega_{X}=\mathcal{O}_{X}(-1)$ (i.e. $X$ is of index 1). Then $X$ does not have (D).

Proof. Recall that $H^{i}\left(X, \mathcal{O}_{X}\right)=0$ for $i>0$, by Serre duality and the Kodaira vanishing theorem; in particular, $X$ is algebraically simply connected, since $\chi\left(X, \mathcal{O}_{X}\right)=1$. Since $b_{2}(X)=1$, we have $\operatorname{Pic}(X)=\mathbb{Z}$, and (11) holds (cf. Lemma 1). Now, Proposition 11 implies that (D) does not hold.

Fano varieties of the above type have been essentially classified by Iskovskih (see [18], [5]). This is extended to positive characteristics in [33].

4.2. (D) and the Point Property. It is useful to consider a property related to (D), which is sometimes a consequence of it.

Let $X$ be a scheme. For a line bundle $\mathcal{L}$ on $X$, we say that the " $\mathcal{L}$-point property holds" if the following is true:

"If for each $x \in X$, there exists a vector bundle $\mathcal{F}$ on $X$ of $\operatorname{rank} d:=\operatorname{dim}(X)$ with $\operatorname{det}(\mathcal{F})=\mathcal{L}$, and a section of $\mathcal{F}$ vanishing exactly at $x$ with multiplicity 1 ."

This implies

$$
c_{1}(\mathcal{F})=c_{1}(\mathcal{L}) \in C H^{1}(X), \quad c_{d}(\mathcal{F})=[x] \in C H^{d}(X) .
$$

Theorem 6. Let $X$ be smooth and proper scheme over an algebraically closed field. Denote by $p_{1}, p_{2}: X \times X \rightarrow X$ the two projections. Suppose that the diagonal property $(D)$ holds, and $\operatorname{Pic}(X)$ is finitely generated. Then there exists a cohomologically trivial line bundle $\mathcal{L}$ on $X$ such that

(i) the $\mathcal{L}^{-1}$-point property holds, and also

(ii) the $\mathcal{L} \otimes \omega_{X}^{-1}$-point property holds.

Proof. Let $\mathcal{E}$ be a rank $\operatorname{dim}(X)$ bundle on $X \times X$ given by (D).

By Lemma 1, the finite generation of $\operatorname{Pic}(X)$ is equivalent to (11). By (11), we have

$$
\operatorname{det}(\varepsilon)=p_{1}^{*} \mathcal{L}_{1} \otimes p_{2}^{*} \mathcal{L}_{2},
$$

for some line bundles $\mathcal{L}_{1}$ and $\mathcal{L}_{2}$ on $X$. Then the restrictions of $\mathcal{E}$ to $X \times\{x\}$ and $\{x\} \times X$ have the determinants $\mathcal{L}_{1}, \mathcal{L}_{2}$ respectively, whatever the choice of $x$. Hence the $\mathcal{L}_{1}$-point property as well as the $\mathcal{L}_{2}$-point property hold for $X$. 
When the determinant of $\mathcal{E}$ does have the above special form (20), then the restriction to the diagonal is $\mathcal{L}_{1} \otimes \mathcal{L}_{2}$, so that, by Theorem 4 , we see that $\mathcal{L}_{1}^{-1}$ and $\mathcal{L}_{2}^{-1}$ are mutually Serre dual line bundles, which are cohomologically trivial line bundles.

Corollary 6. Let $X$ be a smooth and proper scheme over an algebraically closed field, with finitely generated $\operatorname{Pic}(X)$. If for any cohomologically trivial line bundle $\mathcal{L}$ on $X$, either the $\mathcal{L}^{-1}$-point property fails, or the $\mathcal{L} \otimes \omega_{X}^{-1}$-point property fails, then $X$ does not admit $(D)$.

Observe, that there exists a "trivial" instance of the corollary when there are no cohomologically trivial line bundles on $X$ at all.

Proposition 12. A smooth quadric $Q_{3} \subset \mathbb{P}^{4}$ over an algebraically closed field fails to have (D).

Proof. There are two cohomologically trivial line bundles on $Q_{3}: \mathcal{L}_{1}=\mathcal{O}_{Q_{3}}(-1)$ and $\mathcal{L}_{2}=\mathcal{O}_{Q_{3}}(-2)$. Since $\omega_{Q_{3}}=\mathcal{O}_{Q_{3}}(-3)$, we have

$$
\mathcal{L}_{1}^{-1}=\mathcal{L}_{2} \otimes \omega_{Q_{3}}^{-1}
$$

so by Corollary 6 , it suffices to show that the $\mathcal{L}_{1}^{-1}$-point property fails.

We use a standard presentation of the Chow ring $C H^{*}\left(Q_{3}\right)$ as $\mathbb{Z}^{\oplus 4}$ with a suitable ring structure. Let $\left[Q_{2}\right],[L]$, and $[P]$ (quadric surface, line, and point) be the generators of $C H^{1}\left(Q_{3}\right), C H^{2}\left(Q_{3}\right)$, and $C H^{3}\left(Q_{3}\right)$ respectively. There are the following relationships:

$$
\left[Q_{2}\right]^{2}=2[L] \text { and }\left[Q_{2}\right] \cdot[L]=[P] .
$$

If $\mathcal{E}$ is a vector bundle on $Q_{3}$, the total Chern class of $\mathcal{E}$ is of the form

$$
1+d_{1}(\mathcal{E})\left[Q_{2}\right]+d_{2}(\mathcal{E})[L]+d_{3}(\mathcal{E})[P],
$$

where $d_{i}(\mathcal{E}) \in \mathbb{Z}$.

The argument now boils down to showing that there is no rank 3 vector bundle $\mathcal{E}$ on $Q_{3}$ with $d_{3}(\mathcal{E})=1$ and $d_{1}(\mathcal{E})=1$.

Suppose - ad absurdum - that such a bundle exists. We use the formula for the Euler characteristic of $\mathcal{E}$ given by the Grothendieck-Hirzebruch-RiemannRoch theorem (see [9], Example 15.2.5 for a general formula for 3-folds). In fact, 
we use the following explicit version of the formula for smooth quadric 3-folds, given in [7]: for a vector bundle $\mathcal{E}$ on $Q_{3}$,

$$
\chi\left(Q_{3}, \mathcal{E}\right)=\frac{1}{6}\left(2 d_{1}^{3}-3 d_{1} d_{2}+3 d_{3}\right)+\frac{3}{2}\left(d_{1}^{2}-d_{2}\right)+\frac{13}{6} d_{1}+\operatorname{rank}(\mathcal{E}),
$$

where $d_{i}=d_{i}(\mathcal{E})$ are the above numbers. Substituting the present values, we get

$$
\chi\left(Q_{3}, \varepsilon\right)=\frac{15}{2}-2 d_{2} .
$$

This contradicts the fact that $\chi\left(Q_{3}, \mathcal{E}\right)$ is integer, and the proposition has been proved.

Note that since the quadric $Q_{3}$ is birationally isomorphic to the projective 3space which has (D), the property (D) is not a birational invariant in dimension $\geq 3$.

The following result is obtained in a standard way, following the argument that the tangent bundle of a group variety is trivial.

Proposition 13. Let $X$ be a group variety over an algebraically closed field. Then $X$ has (D) if and only if $X$ has the following "weak point property": for some point $x \in X$, there exists a vector bundle $\mathcal{E}$ of rank $\operatorname{dim}(X)$, such that there is a section of $\mathcal{E}$ with zero scheme $x$.

Proof. If $X$ has (D), then for any $x \in X$, the weak point property holds, by restriction of the data giving (D) to $X \times\{x\}$.

Conversely, suppose the weak point property holds with respect to the point $x$. Let $\mathcal{E}$ be the corresponding vector bundle, and $s$ a section with zero scheme $x$. Let $\mu: X \times X \rightarrow X$ be the multiplication, and $i: X \rightarrow X$ the inverse, defining the algebraic group structure on $X$. Define a new map $f: X \times X \rightarrow X$ by

$$
f(u, v)=\mu(\mu(u, i(v)), x) .
$$

Then $f$ is a morphism, whose scheme theoretic fiber $f^{-1}(x)=\Delta_{X}$, the diagonal subscheme. Hence the vector bundle $f^{*} \mathcal{E}$ has the section $f^{*}(s)$ whose zero scheme is the diagonal.

Corollary 7. An abelian variety has (D) precisely if it has the weak point property for some point.

Though we have not resolved whether a smooth cubic 3-fold has (D), we have the following observation, which is a necessary condition for (D), since the only cohomologically trivial line bundle on a cubic 3 -fold $X$ is $\mathcal{O}_{X}(-1)$. 
Lemma 4. A smooth complex cubic 3-fold $X$ has the $\mathcal{O}_{X}(1)$-point property.

Proof. It is known that a smooth cubic 3 -fold $X$ contains lines from the ambient projective space, and is covered by such lines. Further, any line $L \subset X$ has a normal bundle with trivial determinant, from the adjunction formula, since $\omega_{X}=\mathcal{O}_{X}(-2), \omega_{L}=\mathcal{O}_{L}(-2)$, and $\mathcal{O}_{X}(1) \otimes \mathcal{O}_{L}=\mathcal{O}_{L}(1)$ (the last formula holds because $L$ is a line).

If $\mathcal{J}_{L}$ is the ideal sheaf of a line in $X$, then since $H^{i}\left(X, \mathcal{O}_{X}\right)=0, i=1,2$, we have an isomorphism

$$
\operatorname{Ext}^{1}\left(\mathcal{J}_{L}, \mathcal{O}_{X}\right) \cong H^{0}\left(\underline{\operatorname{Ext}}^{1}\left(\mathcal{J}_{L}, \mathcal{O}_{X}\right)\right) \cong \operatorname{Hom}\left(\operatorname{det} \mathcal{J}_{L} / \mathcal{J}_{L}^{2}, \mathcal{O}_{L}\right)=\mathbb{C} .
$$

Thus, from the Serre argument, there is a rank 2 vector bundle $\mathcal{E}$ on $X$ with trivial determinant, together with a section $s$, whose zero scheme is $L$.

Now, suppose $x \in X$ is any point. Choose a line $L \subset X$ passing through $x$, and choose a hyperplane $H$ in $\mathbb{P}^{4}$, not containing $L$, but passing through $x$. Then $L$ and $H$ intersect transversally at $x$. If $t \in H^{0}\left(\mathbb{P}^{4}, \mathcal{O}(1)\right)$ is a section with zero scheme $H$, then the rank 3 vector bundle $\mathcal{F}=\mathcal{E} \oplus \mathcal{O}_{X}(1)$ has a section $\left(s,\left.t\right|_{X}\right)$ with zero scheme $L \cap(H \cap X)=\{x\}$, and the intersection is transverse in $X$. The determinant of $\mathcal{F}$ is clearly $\mathcal{O}_{X}(1)$.

\section{The AFFIne CASE}

It makes sense to ask which smooth affine varieties over an algebraically closed field have (D). If $X=\operatorname{Spec} A$ is a smooth affine $k$-variety of dimension $\leq 2$, then $X$ has (D); in the 2-dimensional case, this follows from Serre's classic argument, since the diagonal is a smooth, codimension 2 subscheme of an affine scheme.

We do have one positive result in the affine case, which may be of interest. This is basically a corollary of work of M. P. Murthy.

Proposition 14. An affine algebraic group over an algebraically closed field has (D).

Proof. If $X$ is an affine algebraic group over an algebraically closed field, then a result of Mohan Kumar and M. P. Murthy (cf. [26], Theorem 3.1) shows that any smooth point is the zero scheme of a section of a vector bundle of rank equal to $\operatorname{dim}(X)$. 
Remark 4. In fact, for an affine algebraic group $X$, we can even say that any point $x \in X$ is a complete intersection (i.e. the vector bundle may be chosen to be a trivial bundle). Hence the diagonal is also a (global) complete intersection. Indeed, Murthy's results in [27] imply that a point on a smooth affine variety is a complete intersection if and only if its class in the Chow group of points vanishes. This holds for $X$ because for unirational smooth affine varieties the Chow group of points is, in fact, zero. One can now naturally ask if the diagonal of any affine algebraic group is a complete intersection in any group-theoretically significant way!

We conjecture that there exist smooth affine complex varieties of any dimension $\geq 3$ for which (D) fails. However, we have been unable to construct such examples. This leads to the following question:

Question: Let $A$ be a smooth affine algebra over an algebraically closed field $k$. Let $K$ be an extension field of $k$ (not necessarily algebraically closed), $A_{K}=$ $A \otimes_{k} K$, and $M \subset A_{K}$ a maximal ideal with residue field $K$. Does there exist a projective $A_{K}$-module $P$ of $\operatorname{rank} n=\operatorname{dim}(A)$ such that there is a surjection $P \rightarrow M ?$

If $K$ is an algebraically closed extension of $k$, this is always true, from Murthy's results. If $X=\operatorname{Spec} A$ satisfies (D), then the question has a positive answer for any field extension $K$. So a negative answer to the question would give a way of constructing counterexamples to (D).

\section{The Topological Diagonal Property}

Let $M$ be a smooth compact connected oriented manifold of real dimension $n$, and let

$$
\Delta \subset M \times M
$$

be the diagonal submanifold. In this section, we introduce topological versions of the diagonal property $(D)$. We also use notation standard in topology (instead of that from algebraic geometry used earlier).

We say that $M$ has property $\left(D_{r}\right)$ if there exists a smooth real vector bundle $\mathcal{E}$ of rank $n$ on $M \times M$ and a smooth section $s$ of $\mathcal{E}$ such that (i) $s$ is transverse to the 0 -section $0_{\mathcal{E}}$ of $\mathcal{E}$ and (ii) $\Delta=s^{-1}\left(0_{\mathcal{E}}\right)$. If further the bundle $\mathcal{E}$ is orientable, we say that $M$ has property $\left(D_{o}\right)$. Finally, if $\operatorname{dim}_{\mathbb{R}} M=2 m$, and $\mathcal{E}$ can be chosen to be a smooth complex vector bundle of $\operatorname{rank}_{\mathbb{C}} \mathcal{E}=m$, we say that $M$ has property $\left(D_{c}\right)$. 
Remark 5. Clearly $\left(D_{o}\right) \Rightarrow\left(D_{r}\right)$. Further, if $M$ satisfies $\left(D_{c}\right)$, then $\varepsilon_{\mid \Delta}$ is isomorphic to the normal bundle of $\Delta$ by the transversality condition, and this normal bundle is known to be isomorphic to the tangent bundle $\tau_{M}$ (cf. (1) in Section 2). It follows that $\mathcal{E}$ being a complex vector bundle forces $\tau_{M}$ to be a complex bundle, so $M$ will have to be an almost complex manifold. $\left(D_{o}\right)$ and $\left(D_{r}\right)$ will follow from $\left(D_{c}\right)$ whenever it holds. Also, if $M$ is a complex manifold, then $(D)$ of the earlier sections will imply $\left(D_{c}\right)$, hence $\left(D_{o}\right)$ and $\left(D_{r}\right)$.

Remark 6. (The topological point property) In analogy with "weak point property" from Section 4 , if $M$ has $\left(D_{r}\right)$, then letting $\mathcal{E}$ be the bundle realizing $\left(D_{r}\right)$, and setting $\mathcal{E}_{1}:=\mathcal{E}_{M \times\{p\}}$, and the section $\sigma:=s_{\mid M \times\{p\}}$ one finds $\sigma \nmid 0_{\mathcal{E}_{1}}$ and $\sigma^{-1}\left(0_{\mathcal{E}_{1}}\right)=\{p\}$. Likewise for $\mathcal{E}_{2}:=\mathcal{E}_{\mid\{q\} \times M}$. We will say that a smooth manifold obeys $\left(P_{r}\right)$ (resp. $\left(P_{o}\right)$ resp. $\left(P_{c}\right)$ ) if there exists a smooth real (resp. real orientable, resp. complex) bundle $\mathcal{H}$ of $\operatorname{rank}_{\mathbb{R}} \mathcal{H}=\operatorname{dim}_{\mathbb{R}} M=n$ (resp. $\operatorname{rank}_{\mathbb{R}} \mathcal{H}=\operatorname{dim}_{\mathbb{R}} M=n$, resp. $\operatorname{rank}_{\mathbb{C}} \mathcal{H}=\operatorname{dim}_{\mathbb{C}} M=m$ ) with a smooth section $\sigma$ meeting $0_{\mathcal{H}}$ transversely at one point. Thus $\left(D_{r}\right)$ (resp. $\left(D_{o}\right)$, resp. $\left.\left(D_{c}\right)\right)$ implies $\left(P_{r}\right)$ (resp. $\left(P_{o}\right)$, resp. $\left.\left(P_{c}\right)\right)$. If $M$ is compact, and $\mathcal{H}$ realizes $\left(P_{r}\right)$ (resp. $\left(P_{o}\right)$, resp. $\left.\left(P_{c}\right)\right)$, then $w_{n}(\mathcal{H})=1\left(\right.$ resp. $e(\mathcal{H})= \pm 1$, resp. $\left.c_{m}(\mathcal{H})= \pm 1\right)$.

For the properties $\left(P_{o}\right)$ and $\left(P_{c}\right)$, we have a converse: if an appropriate bundle $\mathcal{H}$ on the manifold $M$ satisfies $e(\mathcal{H})= \pm 1$ (resp. $\quad c_{m}(\mathcal{H})= \pm 1$ ), and $M$ is connected, then we can use this bundle $\mathcal{H}$ to realize the property $\left(P_{o}\right)$ (resp. $\left(P_{c}\right)$ ). Though this is presumably well known to experts, we sketch a simple argument, since we could not find it in the literature (we thank M. S. Raghunathan for suggesting this argument). First choose a smooth section of $\mathcal{H}$ meeting the zero section transversally; the condition that the Euler/top Chern class is \pm 1 means that, considered as oriented intersections, the numbers of intersections preserving and reversing orientations differ by 1 . If $x, y \in M$ are two points where the chosen section vanishes, and the intersections with the zero section are orientation preserving and reversing respectively, choose a smooth arc from $x$ to $y$ in the manifold, not passing through any other zero of our chosen section (we must have that $\operatorname{dim}_{\mathbb{R}} M>1$, since $M$ supports $\mathcal{H}$ with nontrivial Euler/Chern class). We can find a closed neighborhood of this arc, which again does not contain any other zeroes of our section, and which is diffeomorphic to an $n$-dimensional (resp. $2 m$-dimensional, if $M$ is almost complex) closed disc. 
It then suffices to note that, given an oriented, trivial bundle of rank $n$ (resp. trivial complex bundle of rank $m$ ) on a closed disc $D$, with a section $s$ with precisely two zeroes in $D \backslash \partial D$ as above, the restriction $\left.s\right|_{\partial D}$ has a different extension $\tilde{s}$ as a section over $D$ without any zeroes. Using a trivialization of the bundle, we may view $s$ as a map $s: D \rightarrow V$ to a vector space $V$, with the preimage of the origin being the two points $x, y$; the condition on orientations at $x, y$ imply that $\left.s\right|_{\partial D}: \partial D \rightarrow V \backslash\{0\}$ has degree 0 , and is hence nullhomotopic; hence it extends to a map $\tilde{s}: D \rightarrow V \backslash\{0\}$, which gives the desired section of the bundle without any zeroes on $D$.

Example 1. (Lie Groups) Let $G$ be a Lie group, then $G$ satisfies $\left(D_{r}\right)$ (resp. $\left(D_{o}\right)$, resp. $\left.\left(D_{c}\right)\right)$ iff it satisfies $\left(P_{r}\right)$ (resp. $\left(P_{o}\right)$, resp. $\left(P_{c}\right)$.) The proof is analogous to Proposition 14. Let $\mathcal{H}$ be a bundle realizing $\left(P_{r}\right)$ and a section with a transverse zero at $1 \in G$ (by translating if necessary), and then the bundle $\mathcal{E}:=\mu^{*} \mathcal{H}$, where $\mu: G \times G \rightarrow G$ is $(x, y) \mapsto x y^{-1}$ with pulled back section realizes $\left(D_{r}\right)$. Likewise for $\left(D_{o}\right)$ and also $\left(D_{c}\right)$. It follows that $S^{1}$ satisfies $\left(D_{r}\right)$. Similarly $S O(3)=\mathbb{R P}^{3}$ satisfies $\left(D_{r}\right)$. We will see later that no odd dimensional manifold satisfies $\left(D_{o}\right)$, so $S^{1}$ and $S O(3)$ do not satisfy $\left(D_{o}\right)$.

Example 2. (Products) If two manifolds $M$ and $N$ have $\left(D_{r}\right)$ (resp. $\left(D_{o}\right)$, resp. $\left(D_{c}\right)$, then so does $M \times N$, as remarked in the Introduction, by taking the product of the corresponding bundles. In particular, by the example of $S^{1}$ in Example 1 above, and of $\mathbb{C P}^{1}$ from the next example, manifolds satisfying $\left(D_{r}\right)$ (resp. $\left.\left(D_{c}\right) \Rightarrow\left(D_{o}\right)\right)$ exist in every dimension (resp. every even dimension).

Example 3. (Projective spaces and Grassmannians) For all $k$ the real Grassmannian $G_{k}\left(\mathbb{R}^{n}\right)$ satisfies $\left(D_{r}\right)$, by the construction using the tautological bundles given in the Introduction. Likewise $G_{k}\left(\mathbb{C}^{n}\right)$ satisfies $\left(D_{c}\right)$ by the analogous complex construction. It is not true that an orientable Grassmannian satisfies $\left(D_{o}\right)$, e.g. we shall see below that no odd-dimensional real projective space satisfies $\left(D_{o}\right)$.

Example 4. (Compact Riemann Surfaces) As remarked in the Introduction, if $M$ is a compact connected Riemann surface, then it satisfies $(D)$, and hence $\left(D_{c}\right)$.

Example 5. (The spheres $S^{1}, S^{2}, S^{4}, S^{8}$ ) The circle $S^{1}$, in view of Example 1 (or 3) satisfies $\left(D_{r}\right)$, and $S^{2}=\mathbb{C P}^{1}$ satsifies $\left(D_{c}\right) \Rightarrow\left(D_{o}\right)$ in view of Example 
3. Regarding $S^{4}$ (resp. $S^{8}$ ) as the quaternionic line (resp. octonionic line) and repeating the construction of Example 3 above with respect to the respective tautological bundles defined on these, one checks that $S^{4}$ and $S^{8}$ both satisfy $\left(D_{o}\right)$. They obviously can't satisfy $\left(D_{c}\right)$ because neither is an almost complex manifold (cf. Remark 5).

A Riemannian metric on $M$ induces one on $M \times M$, so metrics result on $\tau_{M \times M}$, and all its subbundles. Let $U$ be a closed $\epsilon$-tubular neighborhood of $\Delta$ in $M \times M$. The tubular neighborhood theorem gives a smooth diffeomorphism $\phi:(U, \partial U) \rightarrow$ $(D(\nu), S(\nu))$, where $D(\nu)$ is the $\epsilon$-disc bundle of the normal bundle $\rho: \nu \rightarrow \Delta$ of $\Delta$ in $M \times M$, and $S(\nu)$ the $\epsilon$-sphere bundle. The map $r:=\rho \circ \phi: U \rightarrow \Delta$ is then a strong deformation retraction of $U$ to its core $\Delta$. Thus there is a bundle diagram

$$
\begin{array}{ccc}
r^{*}(\nu) & \stackrel{D \phi}{\longrightarrow} \rho^{*}(\nu) \\
\rho \downarrow & \downarrow \rho \\
U & \stackrel{\phi}{\longrightarrow} D(\nu) .
\end{array}
$$

The restricted bundle $\rho^{*}(\nu)_{\mid S(\nu)} \rightarrow S(\nu)$ has a tautological section $s$ defined by $v \mapsto v$, which satisfies $\|s(v)\|=\epsilon$ for all $v \in S(\nu)$. Thus we have an orthogonal direct sum decomposition of bundles on $S(\nu)$ :

$$
\rho^{*}(\nu)_{\mid S(\nu)}=\xi \oplus \mathcal{L},
$$

where $\mathcal{L}$ is the trivial line subbundle spanned by $s$ (and $\xi$ denotes its orthogonal complement). It is also well known that $\rho: \nu \rightarrow \Delta$ is isomorphic to the tangent bundle $\rho: \tau_{M} \rightarrow M$ under the identification $\Delta \cong M$, and since $M$ is orientable, so is $\xi$. Hence, under this identification, $\xi$ is isomorphic to the quotient bundle

$$
\rho^{*}\left(\tau_{M}\right) / \mathcal{L} \rightarrow S\left(\tau_{M}\right),
$$

where $\mathcal{L} \rightarrow S\left(\tau_{M}\right)$ is the trivial tautological bundle spanned by the tautological section of $\rho^{*}\left(\tau_{M}\right)$ over $S\left(\tau_{M}\right)$.

Let $\mathcal{F}:=\phi^{*}(\xi)$, a rank $(n-1)$ subbundle of $r^{*}(\nu)_{\mid \partial U}$. It is isomorphic to the rank $(n-1)$ bundle $\rho^{*}\left(\tau_{M}\right) / \mathcal{L} \rightarrow S\left(\tau_{M}\right)$ under the above identifications. Note that $\mathcal{F}$ is an orientable bundle on $\partial U$.

Remark 7. The restriction of the bundle $\xi$ above to each fiber $S\left(\nu_{x}\right)$ of the sphere bundle $\rho: S(\nu) \rightarrow M$ is the tangent bundle $\tau_{n-1}$ of the sphere $S\left(\nu_{x}\right)$. Consequently, the bundle $\mathcal{F}$, when restricted to a fiber $r^{-1}(x)$ of the fiber bundle $r: \partial U \rightarrow \Delta$, is isomorphic to $\tau_{n-1}$. This is clear, since the fiber of $\mathcal{L}$ at a point $v \in S\left(\nu_{x}\right)$ is precisely $\mathcal{L}_{v}=\mathbb{R} v$. 
Since the $n=1$ (i.e. $S^{1}$ ) case is completely settled by Example 1, we will assume henceforth that $n=\operatorname{dim}_{\mathbb{R}} M \geq 2$. The following lemma is the key technical result of this section. We shall write $U^{\circ}$ for the interior of $U$.

Lemma 5. Let $M, U, \Delta$, be as above. Set $X:=(M \times M) \backslash U^{\circ}$. Then $M$ has $\left(D_{r}\right)$ iff the rank $(n-1)$ bundle $\mathcal{F} \rightarrow \partial U$ defined above is isomorphic to the restriction to $\partial U=\partial X$ of a smooth rank $(n-1)$ bundle $\mathcal{G}$ on $X$. Further, $M$ has $\left(D_{o}\right)$ iff the bundle $\mathcal{G}$ can be chosen to be orientable.

These are both problems in homotopy theory, of extending classifying maps $\partial X \rightarrow B G$ for the bundle $\mathcal{F}$ (where $G=O(n-1)$ for $\left(D_{r}\right)$, resp. $S O(n-1)$ for $\left.\left(D_{o}\right)\right)$ to $X$.

Proof. We first prove the "only if" part. Suppose there exists a rank $n$ smooth real vector bundle $\pi: \mathcal{E} \rightarrow M \times M$, and $s$ a smooth section transverse to the zero-section $0_{\mathcal{E}}$ such that the diagonal $\Delta=s^{-1}\left(0_{\mathcal{E}}\right)$. The strong deformation retraction $r: U \rightarrow \Delta$ makes $\mathcal{E}_{\mid U}$ isomorphic to $r^{*}\left(\mathcal{E}_{\mid \Delta}\right)$, so $\mathcal{E}_{r(y)}$ is identified with $\varepsilon_{y}$ for all $y \in U$. The section $s$ is nowhere vanishing on

$$
X=(M \times M) \backslash U^{\circ},
$$

and hence defines a trivial line subbundle $\Lambda$ of $\mathcal{E}_{\mid X}$, and a splitting of bundles on $X$ :

$$
\mathcal{E}_{\mid X}=\mathcal{G} \oplus \Lambda
$$

where $\mathcal{G}$ is a rank $(n-1)$ bundle on $X$. Using the transversality condition on $s$, the tubular neighborhood theorem, the inverse function theorem and compactness of $M$, and choosing $\epsilon$ (the radius of $U$ ) small enough, it is easy to see that there is a smooth bundle equivalence of $\pi: \mathcal{E}_{\mid U} \rightarrow U$ and $\rho: r^{*}\left(\tau_{\Delta}\right)_{\mid U} \rightarrow U$ which carries the section $s$ of the former to the tautological section of the latter ( $U$ being identified with the $\epsilon$-disc bundle $D\left(\tau_{\Delta}\right)=D\left(\tau_{M}\right)=D(\nu)$ ). Thus on $\partial U=\partial X$, quotienting by the respective trivial line bundles defined by these sections, it follows that $\mathcal{G}_{\mid \partial U}=\mathcal{G}_{\mid \partial X}$ is isomorphic to $\mathcal{F}$. If $M$ had $\left(D_{o}\right)$, and $\mathcal{E}$ was an orientable bundle realizing $\left(D_{o}\right)$ to begin with, then $\mathcal{G}=\mathcal{E}_{\mid X} / \epsilon_{X}^{1}$ would also be orientable. This proves the "only if" parts of both the first and second statements.

For the "if" part, let $\mathcal{G}$ be given on $X$ as in the statement. Construct the bundle $\mathcal{E}$, by taking $r^{*}(\nu)$ on $U$, and gluing it to the bundle $\mathcal{G} \oplus \epsilon_{X}^{1}$ on $X$, after ensuring that the decomposition $r^{*}(\nu)=\mathcal{F} \oplus \phi^{*}(\mathcal{L})$ on $\partial U$ is preserved, viz. the first summand $\mathcal{G}_{\mid \partial U}$ is glued to the first summand $\mathcal{F}_{\mid \partial U}$ via the given isomorphism, 
and the second trivial summand $\epsilon_{X}^{1}$ is glued to the second trivial summand $\phi^{*} \mathcal{L}_{\mid \partial U}$ on $\partial U$ by matching the section $\sigma$ of the trivial bundle $\phi^{*} \mathcal{L} \rightarrow \partial U$ defined above with any nowhere vanishing section of $\epsilon_{X}^{1}$ which extends $\sigma$ to $X$ (this is possible, since $n \geq 2$ implies $\partial X$ is connected). The section $\sigma$ of $r^{*}(\nu) \rightarrow \partial U$ above is the restriction of the tautological section, also denoted $\sigma$, of $r^{*}(\nu) \rightarrow U$ which is transverse to the zero section. Thus the matched section $s$ of the whole bundle $\mathcal{E}$ vanishes exactly on $\Delta$, with $s \nmid 0_{\mathcal{E}}$.

The lemma has been proved.

We have an analogue of this lemma for $\left(D_{c}\right)$. Assume (in view of Remark 5) that $M$ is an almost complex manifold of real dimension $n=2 m$. Then in the notation of the last section, the normal bundle $\nu \cong \tau_{M}$ of $\Delta$ is a complex vector bundle, and $r^{*}(\nu) \rightarrow \partial U$ splits off a complex line subbundle $\epsilon_{c}^{1}$ defined by the complex span of the tautological section of $r^{*}(\nu)_{\mid \partial U}$. Thus we may write

$$
r^{*}(\nu)_{\mid \partial U}=\mathcal{F}_{c} \oplus \epsilon_{c}^{1},
$$

where $\mathcal{F}_{c}$ is now a complex vector bundle on $\partial U=\partial X$ with $\operatorname{rank}_{\mathbb{C}} \mathcal{F}_{c}=m-1$.

Lemma 6. Let $M$ be an almost complex manifold of $\operatorname{dim}_{\mathbb{C}} M=m$. Then $M$ has $\left(D_{c}\right)$ iff the bundle $\mathcal{F}_{c}$ of complex rank $m-1$ on $\partial X$ is isomorphic as a complex bundle to the restriction of a complex vector bundle $\mathcal{G}_{c}$ on $X$. This is again a homotopy problem as in Lemma 5, with structure group $G=U(m-1)$.

Proof. The proof is a minor modification of that of Lemma 5, and is therefore omitted.

Corollary 8. (Compact Riemann surfaces again) Let $M$ be a compact Riemann surface. Then $M$ has $\left(D_{c}\right)$.

Proof. We saw this in Example 4. It also immediately follows from Lemma 6, since $\mathcal{F}_{c}$ is a complex vector bundle of rank 0 (!)

Remark 8. Lemmas 5, 6 allow us to identify the obstructions to $\left(D_{r}\right),\left(D_{o}\right)$ and $\left(D_{c}\right)$ as some relative cohomology classes of the pair $(X, \partial X)$ with local coefficients in $\pi_{i}(B G)$, where $G=O(n), S O(n), U(n)$ respectively. Since these are not computable obstructions, we skip the details.

Theorem 7. The sphere $S^{n}$ has $\left(D_{r}\right)$ if and only if $n=1,2,4$ or 8 . (All except the first have $\left(D_{o}\right)$.) 
Proof. The unit sphere bundle $S\left(\tau_{n}\right)$ is the Stiefel manifold $V_{2}\left(\mathbb{R}^{n+1}\right)$ of orthonormal 2-frames $(x, v)$ in $\mathbb{R}^{n+1}$. The bundle projection $\rho: S\left(\tau_{n}\right) \rightarrow S^{n}$ is projection into the first factor, and thus we have the spherical fiber bundle

$$
S_{x}^{n-1} \stackrel{j_{x}}{\rightarrow} V_{2}\left(\mathbb{R}^{n+1}\right) \stackrel{\rho}{\rightarrow} S^{n}
$$

with fiber $S_{x}^{n-1}=\rho^{-1}(x)$ over $x$. The tautological section of the bundle

$$
\rho^{*}\left(\tau_{n}\right) \rightarrow V_{2}\left(\mathbb{R}^{n+1}\right)
$$

is the map $(x, v) \mapsto v$. Set $\epsilon=\pi$ (length of a semicircle), and denote the closed $\pi$-tubular neighborhood of $\Delta$ by $U$ as above. The complement

$$
X=\left(S^{n} \times S^{n}\right) \backslash U^{\circ}
$$

is a closed $\pi$-tubular neighborhood of the antidiagonal $\Gamma$, which is the graph of the antipodal map $A: S^{n} \rightarrow S^{n}$ defined by $A x=-x$.

The involution $1 \times A$ smoothly identifies $U$ with $X$. The common boundary $\partial U=\partial X$ is diffeomorphic to the $\pi$-sphere bundle $S_{\pi}\left(\tau_{n}\right)=V_{2}\left(\mathbb{R}^{n+1}\right)$. Also, $X$ becomes a disc bundle $D_{\pi}\left(\nu_{\Gamma}\right)=D_{\pi}\left(\tau_{n}\right)$, and contains the antidiagonal $\Gamma$ as a strong deformation retract. Let $\theta: X \rightarrow \Gamma$ denote the retraction, coming from the bundle projection

$$
\nu_{\Gamma} \cong \tau_{n} \rightarrow \Gamma .
$$

Because $\theta$ is a deformation retraction, every bundle $\mathcal{G}$ on $X$ is the $\theta$-pullback of a bundle on $\Gamma$, equivalently a $\rho$-pullback of a bundle on $S^{n}$. Hence, it follows from Lemma 5 that $S^{n}$ has $\left(D_{r}\right)$ iff the bundle $\mathcal{F} \rightarrow V_{2}\left(\mathbb{R}^{n+1}\right)$ is isomorphic to the pullback under $\rho$ of some bundle on $S^{n}$. By (24),

$$
\rho \circ j_{x}: S_{x}^{n-1} \rightarrow S^{n}
$$

is the constant map to $x$, it follows that the $\rho$-pullback of any bundle $\mathcal{G}$ on $S^{n}$ will be trivial when restricted to a fiber $S_{x}^{n-1}$. It follows that $\mathcal{F}_{\mid S_{x}^{n-1}}$ is isomorphic to a trivial bundle on $S_{x}^{n-1}$. By Remark $7, \mathcal{F}_{\mid S_{x}^{n-1}}$ is isomorphic to the tangent bundle $\tau_{n-1}$ of $S_{x}^{n-1}$. Hence, $\mathcal{F}$ will be a $\rho$-pullack of a bundle on $S^{n}$ only if $\tau_{n-1}$ is trivial. Hence $n-1=0,1,3$, or 7 by [22], Theorem 2. This proves the "only if" part of the theorem. For the "if" part, cf. Example 5.

The theorem has been proved.

All homologies and cohomologies hereafter are with $\mathbb{Z}$ coefficients unless otherwise stated. 
Theorem 8. Let $M$ be a compact orientable manifold of odd dimension. Then $M$ does not have $\left(D_{o}\right)$. If further $H_{1}\left(M, \mathbb{Z}_{2}\right)=0$, then it does not have $\left(D_{r}\right)$.

Proof. Let $\mathcal{E}$ be an orientable bundle of odd real rank $2 k+1$ on $M \times M$, where $\operatorname{dim}_{\mathbb{R}} M=2 k+1$. It is well-known that the Euler class $e\left(\mathcal{E}_{1}\right)$ of $\mathcal{E}_{1}:=\mathcal{E}_{\mid M \times\{p\}}$ must be zero, since $\varepsilon_{1}$ is of odd rank $=2 k+1=\operatorname{dim}_{\mathbb{R}} M$ and top homology of $M$ is $\mathbb{Z}$, devoid of 2 -torsion. This contradicts property $\left(P_{o}\right)$ of Remark 6 , so $M$ doesn't satisfy $\left(D_{o}\right)$.

For the second assertion, note that if $H_{1}\left(M, \mathbb{Z}_{2}\right)=0$, then $H^{1}\left(M, \mathbb{Z}_{2}\right)=0$ and also $H^{1}\left(M \times M, \mathbb{Z}_{2}\right)=0$. In particular, every bundle on $M \times M$ is orientable. Thus if $M$ satisfies $\left(D_{r}\right)$, it automatically satisfies $\left(D_{o}\right)$. But this contradicts the first statement.

Remark 9. We note from Example 3 of $\mathbb{R P}^{3}$ (satisfying $\left(D_{r}\right)$ but not $\left(D_{o}\right)$ ) above that this is a sharp result, i.e. the $H_{1}\left(M, \mathbb{Z}_{2}\right)=0$ condition cannot be dropped. Note that Theorem 7 for spheres of odd dimension $n \geq 2$ follows from the last theorem.

Theorem 9. Let $M$ be an almost complex manifold of $\operatorname{dim}_{\mathbb{C}} M=2$. Then $M$ has $\left(D_{c}\right)\left(\Rightarrow\left(D_{o}\right) \Rightarrow\left(D_{r}\right)\right)$. (This is in contrast with the results of Section 3 on surfaces in the algebraic setting.)

Proof. We appeal to Lemma 6 . The bundle $\mathcal{F}_{c}$ on $\partial X$ defined there is a complex line bundle, and hence it extends to $X$ iff its first Chern class $c_{1}\left(\mathcal{F}_{c}\right) \in H^{2}(\partial X)$ lifts to $H^{2}(X)$. So it is enough to show that the restriction homomorphism

$$
H^{2}(X) \rightarrow H^{2}(\partial X)
$$

is surjective. From the commutative diagram induced by inclusions

$$
\begin{array}{cc}
H^{2}(X) & \rightarrow H^{2}(\partial X) \\
j^{*} \uparrow & \uparrow l^{*} \\
H^{2}(M \times M) & \rightarrow H^{2}(U)
\end{array}
$$

we note that the bottom restriction map is the same as the map

$$
H^{2}(M \times M) \stackrel{\delta^{*}}{\rightarrow} H^{2}(M)
$$

(by deforming $U$ to its core $\Delta$ ) which is a split surjection. (Recall that $\delta$ denotes the diagonal embedding.) The left vertical map is an isomorphism, for by excision

$$
H^{i}(M \times M, X) \cong H^{i}(D(\nu), S(\nu))
$$


and this vanishes for $0 \leq i \leq 3$ by the Thom isomorphism $\left(\nu \cong \tau_{M}\right.$ is a bundle of real rank 4). Also, the right vertical arrow is an isomorphism because

$$
H^{i}(U, \partial X)=H^{i}(U, \partial U)=H^{i}(D(\nu), S(\nu))=0
$$

for $0 \leq i \leq 3$, again by the Thom isomorphism.

Hence the top horizontal map is a surjection, and the assertion follows.

Lemma 7 (cf. [1]). Let $M$ be an almost complex manifold with $\operatorname{dim}_{\mathbb{C}} M=3$. Let $\mathcal{E}$ be a smooth complex vector bundle on $M$ of any rank, with Chern classes $c_{i}(\mathcal{E}) \in H^{2 i}(M, \mathbb{Z})$, for $1 \leq i \leq 3$. Then these Chern classes satisfy the following identity:

$$
c_{3}(\mathcal{E})-c_{2}(\mathcal{E})\left(c_{1}(M)+c_{1}(\mathcal{E})\right)=2 m \mu
$$

for some $m \in \mathbb{Z}$, where $\mu \in H^{6}(M, \mathbb{Z}) \cong \mathbb{Z}$ is a generator.

Proof. Cf. [1] for details. We remark that even though this result is stated there for complex manifolds, the same proof works by appealing to the generalized Riemann-Roch for almost complex manifolds. Specifically, that the Todd characteristic class $T(M, \mathcal{E})$ is integral (cf. Theorem 25.5.4 in Appendix 1 of [17].)

Theorem 10. Let $M$ be an almost complex manifold of $\operatorname{dim}_{\mathbb{C}} M=3$. Assume that $H^{1}(M, \mathbb{Z})=0$ and $H^{2}(M, \mathbb{Z})=\mathbb{Z}$. Then if $M$ satisfies $\left(D_{c}\right)$, the second Stiefel-Whitney class $w_{2}(M)$ vanishes (i.e. $M$ is spin).

Proof. Let $\mathcal{E}$ be a smooth complex rank 3 bundle on $M \times M$, realizing $\left(D_{c}\right)$. Let $x$ denote a generator of $H^{2}(M, \mathbb{Z})$. Since $H^{1}(M, \mathbb{Z})=0$, and $H^{2}(M, \mathbb{Z})=\mathbb{Z} x$, we have by the Künneth formula that

$$
H^{2}(M \times M)=\mathbb{Z}(x \times 1) \oplus \mathbb{Z}(1 \times x),
$$

where $\times$ denotes the cohomology cross product. Hence the first Chern class of $\mathcal{E}$ is given by

$$
c_{1}(\mathcal{E})=a_{1}(x \times 1)+a_{2}(1 \times x) \in H^{2}(M \times M, \mathbb{Z})
$$

with $a_{1}, a_{2} \in \mathbb{Z}$. Since $\mathcal{E}$, restricted to the diagonal $\Delta$, is isomorphic as a complex vector bundle to the normal bundle $\nu$ of $\Delta$, i.e. $\tau_{M}$, it follows that

$$
\delta^{*}\left(c_{1}(\mathcal{E})\right)=a_{1}(x .1)+a_{2}(1 . x)=\left(a_{1}+a_{2}\right) x=c_{1}(M) .
$$


Thus we have the relation (analogous to the "weak point property" from Section 4)

$$
\left(a_{1}+a_{2}\right) x=c_{1}(M)
$$

We noted in Remark 6, that the restriction of $\mathcal{E}$ to the slices $M \times\{p\}$ and $\{q\} \times M$ will have Euler class \pm 1 times the generator. Thus the 3rd Chern classes of the rank 3 bundles $\mathcal{E}_{1}:=\mathcal{E}_{\mid M \times\{p\}}$ and $\mathcal{E}_{2}:=\mathcal{E}_{\mid\{q\} \times M}$ are both equal to $\pm \mu$, where $\mu$ is the fundamental class in $H^{6}(M, \mathbb{Z})$. Clearly $c_{1}\left(\mathcal{E}_{1}\right)=a_{1} x$ and $c_{1}\left(\mathcal{E}_{2}\right)=a_{2} x$. From Lemma 7, applied to $\mathcal{E}_{1}$ and Eq. (26), it follows that

$$
c_{2}\left(\mathcal{E}_{1}\right)\left(c_{1}(M)+c_{1}\left(\mathcal{E}_{1}\right)\right)=c_{2}\left(\mathcal{E}_{1}\right)\left(2 a_{1}+a_{2}\right) x=c_{3}\left(\mathcal{E}_{1}\right)+2 m_{1} \mu=\left(2 m_{1} \pm 1\right) \mu
$$

and similarly

$$
c_{2}\left(\mathcal{E}_{2}\right)\left(2 a_{2}+a_{1}\right) x=\left(2 m_{2} \pm 1\right) \mu
$$

for some $m_{i} \in \mathbb{Z}$. Reading these relations in $\mathbb{Z}_{2}$-cohomology, we find

$$
a_{1} \equiv a_{2} \equiv 1 \quad \bmod 2 \text {. }
$$

Thus $a_{1}+a_{2} \equiv 0 \bmod 2$. By Eq. (26), it follows that modulo 2

$$
w_{2}(M)=c_{1}(M)=0 .
$$

The theorem has been proved.

Remark 10. The condition $H^{2}(M, \mathbb{Z})=\mathbb{Z}$ cannot be dropped in Theorem 10 . E.g., we know that $\left(D_{c}\right)$ holds for $\mathbb{C P}^{2} \times \mathbb{C P}^{1}$ (by Examples 2 and 3 ) which is not spin. This condition seems to be the topological analogue of the condition $\operatorname{Pic}(X)=\mathbb{Z}$ in the algebraic theory. We also note that the converse to Theorem 10 would also be false. It is known that $S^{6}$ is an almost complex manifold, which is clearly spin, but cannot satisfy $\left(D_{c}\right)$ since it does not satisfy $\left(D_{r}\right)$ by Theorem 7 above.

Corollary 9. Let $M \subset \mathbb{C P}^{N}$ be a smooth projective variety of $\operatorname{dim}_{\mathbb{C}} M=3$. Assume that $M$ is a strict complete intersection (alternatively, a set-theoretically complete intersection with $\left.H_{1}(M, \mathbb{Z})=0\right)$. Then $M$ has $\left(D_{c}\right)$ only if $M$ is spin.

Proof. If $M$ is a smooth projective set-theoretically complete intersection of complex dimension 3, it is known (cf. Cor. 7.6 on p. 149 of [16]) that for such an $M$ we have

$$
H^{1,0}(M)=H^{0,1}(M)=0 .
$$


By Hodge decomposition, it follows that $H^{1}(X, \mathbb{Z})=0$. The same result quoted above shows that

$$
H^{0,2}(M)=H^{2,0}(M)=0 \quad \text { and } \quad H^{1,1}(M)=\mathbb{C} .
$$

It follows again that $H^{2}(M, \mathbb{C})=\mathbb{C}$. Since by hypothesis $H_{1}(X, \mathbb{Z})=0$, it follows that $H^{2}(X, \mathbb{Z})$ has no torsion, equals $\mathbb{Z}$, and is generated by the hyperplane class. Similarly, for a strict smooth complete intersection $M$ with $\operatorname{dim}_{\mathbb{C}} M \geq 3$, it is known that $H^{2}(M, \mathbb{Z})=\mathbb{Z}$ by the Grothendieck-Lefschetz theorem (cf., e.g., [16]). The result now follows from Theorem 10 .

Corollary 10. Let $M$ be a smooth strict complete intersection of $\operatorname{dim}_{\mathbb{C}} M=3$ in $\mathbb{C P}^{n}$, with $M=X_{1} \cap \cdots \cap X_{n-3}$ with $X_{i}$ smooth hypersurfaces of degree $d_{i}$. Then $M$ has $\left(D_{c}\right)$ only if

$$
n+1-\sum_{i} d_{i}
$$

is even. In particular, a smooth hypersurface $M$ in $\mathbb{C P}^{4}$ has $\left(D_{c}\right)$ only if it is of odd degree. Thus, a smooth quadric 3 -fold in $\mathbb{C P}^{4}$ does not have $\left(D_{c}\right)$.

Proof. It is known that the first Chern class of the tangent bundle of $M$ is $(n+$ $\left.1-\sum_{i} d_{i}\right)$ times the hyperplane class (indeed, the normal bundle of $M$ in $\mathbb{C P}^{n}$ is $\left.\oplus_{i} \mathcal{O}\left(d_{i}\right)\right)$. Thus, $c_{1}(M)$ is an even multiple of the hyperplane class if and only if the number (27) is even. Thus, $M$ is spin if and only if this number is even, and the previous corollary implies the result.

When $n=4$, and $M$ is a hypersurface, the number (27) is even if and only if $d$ is odd. In particular, a smooth quadric in $\mathbb{C P}^{4}$ does not have $\left(D_{c}\right)$ (compare with Proposition 12 from Section 4).

We remark that since the quadrics of complex dimension 1 and 2 are respectively $\mathbb{C P}^{1}$ and $\mathbb{C P}^{1} \times \mathbb{C P}^{1}$, they both satisfy $\left(D_{c}\right)$. The quadric of dimension 3 does not satisfy $\left(D_{c}\right)$ by Corollary 10 . This last fact will now be generalised to all smooth projective quadric hypersurfaces of odd complex dimension $\geq 3$.

Up to the end of the proof of Theorem 11 , we shall now write $\mathbb{P}^{n}$ for $\mathbb{C P}^{n}$.

Proposition 15. Let $Q_{2 m-1} \subset \mathbb{P}^{2 m}$ denote the smooth odd-dimensional quadric hypersurface $V\left(X_{0}^{2}+\cdots+X_{2 m}^{2}\right)$, and let $m \geq 2$. Then the integral cohomology ring of $Q_{2 m-1}$ is given by

$$
H^{*}\left(Q_{2 m-1}\right)=\mathbb{Z}[x, y] /\left\langle x^{m}-2 y, y^{2}\right\rangle,
$$


where $x:=c_{1}\left(\mathcal{O}_{Q_{2 m-1}}(1)\right)$ is the generator of $H^{2}\left(Q_{2 m-1}\right)$, and $y$ is the generator of $H^{2 m}\left(Q_{2 m-1}\right)$. In particular,

$$
\begin{aligned}
H^{2 k+1}\left(Q_{2 m-1}\right) & =0 \text { for all } k \\
H^{2 k}\left(Q_{2 m-1}\right) & =\mathbb{Z} x^{k} \text { for all } 0 \leq k \leq m-1 \\
& =\mathbb{Z} x^{k-m} y \text { for all } m \leq k \leq 2 m-1 .
\end{aligned}
$$

Proof. This result is well-known, but we sketch the proof for completeness. There is an inclusion $j: \mathbb{P}^{m-1} \hookrightarrow Q_{2 m-1}$, where $\mathbb{P}^{m-1}$ is the linear subspace of $\mathbb{P}^{2 m}$ defined by

$\left\{\left[x_{0}: x_{1}: \cdots: x_{2 m}\right] \in \mathbb{P}^{2 m}: x_{0}+\sqrt{-1} x_{1}=\cdots=x_{2 m-2}+\sqrt{-1} x_{2 m-1}=x_{2 m}=0\right\}$.

Letting $i: Q_{2 m-1} \hookrightarrow \mathbb{P}^{2 m}$ denote the natural inclusion we have the composite homomorphisms

$$
\begin{array}{r}
H^{r}\left(\mathbb{P}^{2 m}\right) \stackrel{i^{*}}{\rightarrow} H^{r}\left(Q_{2 m-1}\right) \stackrel{j^{*}}{\rightarrow} H^{r}\left(\mathbb{P}^{m-1}\right), \\
H_{r}\left(\mathbb{P}^{m-1}\right) \stackrel{j_{*}}{\rightarrow} H_{r}\left(Q_{2 m-1}\right) \stackrel{i_{*}}{\rightarrow} H_{r}\left(\mathbb{P}^{2 m}\right)
\end{array}
$$

which are isomorphisms for $0 \leq r \leq 2 m-2$, since $i \circ j$ is a linear inclusion. It follows that $H^{r}\left(Q_{2 m-1}\right)=H_{r}\left(Q_{2 m-1}\right)=0$ for $r$ odd and $0 \leq r \leq 2 m-2$. Furthermore, $H^{2 k}\left(Q_{2 m-1}\right)=\mathbb{Z} x^{k}$ for $0 \leq k \leq m-1$. By Poincaré duality on $Q_{2 m-1}$, we have $H^{i}\left(Q_{2 m-1}\right)=0$ for all odd $i, 0 \leq i \leq 4 m-2$. Similarly,

$$
j_{*}: H_{2 k}\left(\mathbb{P}^{m-1}\right) \rightarrow H_{2 k}\left(Q_{2 m-1}\right)
$$

is an isomorphism of infinite cyclic groups for $0 \leq k \leq m-1$.

Setting $D_{i}, i=1,2$, to be the Poincaré duality isomorphisms for $\mathbb{P}^{m-1}$ and $Q_{2 m-1}$ respectively, it follows by the preceding paragraph that the composition

$$
\begin{aligned}
& H^{2 k}\left(\mathbb{P}^{m-1}\right) \stackrel{D_{1}}{\rightarrow} H_{2 m-2-2 k}\left(\mathbb{P}^{m-1}\right) \stackrel{j_{*}}{\rightarrow} H_{2 m-2-2 k}\left(Q_{2 m-1}\right) \\
& \stackrel{D_{2}^{-1}}{\rightarrow} H^{2 m+2 k}\left(Q_{2 m-1}\right)
\end{aligned}
$$

is an isomorphism for $0 \leq k \leq m-1$. This composite map is the integral cohomology Gysin homomorphism denoted $j_{\text {! }}$; so, setting $k=0$, we find that $H^{2 m}\left(Q_{2 m-1}\right)$ is a cyclic group generated by $y=j ! 1$. Also, $j$ ! is a $H^{*}\left(Q_{2 m-1}\right)$ module homomorphism, so $H^{2 m+2 k}\left(Q_{2 m-1}\right)$ is a cyclic group generated by

$$
j_{!}\left(h^{k}\right)=x^{k} j ! 1=x^{k} y
$$


for all $0 \leq k \leq m-1$. Since $H^{4 m}\left(Q_{2 m-1}\right)=0$, it follows that $y^{2}=0$. Since $Q_{2 m-1}$ is a degree 2 hypersurface in $\mathbb{P}^{2 m}$, we have

$$
\left\langle x^{2 m-1},[Q]\right\rangle=2,
$$

where $[Q] \in H_{4 m-2}\left(Q_{2 m-1}\right)$ is the fundamental homology class of $Q_{2 m-1}$. Thus

$$
\left\langle x^{m} \cdot x^{m-1},[Q]\right\rangle=2 .
$$

By Poincaré duality the generators $y$ of $H^{2 m}$ and $x^{m-1}$ of $H^{2 m-2}$ are dually paired, so we have

$$
\left\langle y \cdot x^{m-1},[Q]\right\rangle=1 .
$$

Thus $x^{m}=2 y$ and the proposition is proved.

Corollary 11. The cohomology ring $H^{*}\left(Q_{2 m-1}, \mathbb{Z}_{2}\right)$ (where $\left.m \geq 2\right)$ is given by

$$
H^{*}\left(Q_{2 m-1}, \mathbb{Z}_{2}\right)=\mathbb{Z}_{2}[\xi, \eta] /\left\langle\xi^{m}, \eta^{2}\right\rangle,
$$

where $\xi$ (resp. $\eta$ ) is the mod 2 reduction of $x$ (resp. y) of the last proposition. Alternatively, $\xi=w_{2}\left(\mathcal{O}_{Q_{2 m-1}}(1)\right)$, the second Stiefel-Whitney class of the canonical bundle on $Q_{2 m-1}$ considered as a real 2-plane bundle, and $\eta=j ! 1$, where

$$
j_{!}: H^{*}\left(\mathbb{P}^{m-1}, \mathbb{Z}_{2}\right) \rightarrow H^{*+2 m}\left(Q_{2 m-1}, \mathbb{Z}_{2}\right)
$$

is the $\mathbb{Z}_{2}$-cohomology Gysin homomorphism. In particular,

$$
\begin{aligned}
H^{2 k+1}\left(Q_{2 m-1}, \mathbb{Z}_{2}\right) & =0 \text { for all } k ; \\
H^{2 k}\left(Q_{2 m-1}, \mathbb{Z}_{2}\right) & =\mathbb{Z}_{2} \xi^{k} \text { for all } 0 \leq k \leq m-1 \\
& =\mathbb{Z}_{2} \xi^{k-m} \eta \text { for all } m \leq k \leq 2 m-1 .
\end{aligned}
$$

Proof. The assertion is immediate from the last proposition. For a complex vector bundle, the total Stiefel-Whitney class is the mod 2 reduction of the total Chern class, so $\xi=w_{2}\left(\mathcal{O}_{Q_{2 m-1}}(1)\right)$.

Lemma 8. Let $m \geq 2$. Then the second Steenrod squaring operation $S q^{2}$ on $H^{*}\left(Q_{2 m-1}, \mathbb{Z}_{2}\right)$ satisfies the relations

$$
\begin{aligned}
S q^{2}(\xi) & =\xi^{2} \\
S q^{2}(\eta) & =(m-1) \xi \eta \quad \bmod 2 \\
S q^{2}\left(\xi^{m-2} \eta\right) & =\xi^{m-1} \eta \quad \bmod 2,
\end{aligned}
$$

where $\xi$ and $\eta$ are the algebra generators from Corollary 11. 
Proof. Since $S q^{i} x=x^{2}$ for $x \in H^{i}$ (cf. [23], part (3) on p. 90), and $\xi \in$ $H^{2}\left(Q_{2 m-1}, \mathbb{Z}_{2}\right)$, it follows that $S q^{2}(\xi)=\xi^{2}$.

For the second formula, one notes that the Gysin homomorphism $j$ ! is well known to be the composition

$$
\begin{aligned}
& H^{i}\left(\mathbb{P}^{m-1}, \mathbb{Z}_{2}\right) \stackrel{\phi}{\rightarrow} H^{i+2 m}\left(D(\nu), S(\nu) ; \mathbb{Z}_{2}\right) \\
& \stackrel{\left(l^{*}\right)^{-1}}{\rightarrow} H^{i+2 m}\left(Q_{2 m-1}, Q_{2 m-1} \backslash \mathbb{P}^{m-1} ; \mathbb{Z}_{2}\right) \rightarrow H^{i+2 m}\left(Q_{2 m-1}, \mathbb{Z}_{2}\right),
\end{aligned}
$$

where $\nu$ is the real rank $2 m$ normal bundle of $\mathbb{P}^{m-1}$ in $Q_{2 m-1}, D(\nu)$ its disc bundle, $S(\nu)$ its sphere bundle, $\phi$ the $\mathbb{Z}_{2}$ Thom isomorphism for $\nu,\left(l^{*}\right)^{-1}$ is an excision isomorphism, and the last arrow is restriction. For brevity's sake, denote the composite of the last two maps by $\alpha$. Then

$$
\eta=j_{!} 1=\alpha(\phi(1))=\alpha\left(U_{\nu}\right),
$$

where $U_{\nu} \in H^{2 m}\left(D(\nu), S(\nu) ; \mathbb{Z}_{2}\right)$ is the $\mathbb{Z}_{2}$ Thom class of $\nu$. Since $\alpha$ is the composite of maps induced by restriction (and the inverse of a restriction), the functorial operation $S q^{2}$ commutes with $\alpha$. Thus

$$
S q^{2}(\eta)=S q^{2}\left(\alpha\left(U_{\nu}\right)\right)=\alpha\left(S q^{2} U_{\nu}\right)
$$

It remains to determine $S q^{2} U_{\nu}$. By Thom's identity for Stiefel-Whitney classes (p. 91, (loc.cit.)), we have $\phi\left(w_{i}(\mathcal{E})\right)=S q^{i} U_{\mathcal{E}}$ for any real bundle $\mathcal{E}$, so $S q^{2} U_{\nu}=$ $\phi\left(w_{2}(\nu)\right)$. The normal bundle of $Q_{2 m-1}$ in $\mathbb{P}^{2 m}$ is $\mathcal{O}_{Q_{2 m-1}}(2)$, and the normal bundle of the linear subspace $\mathbb{P}^{m-1}$ in $\mathbb{P}^{2 m}$ is the sum of $(m+1)$ copies of $\mathcal{O}_{\mathbb{P}^{m-1}}(1)$, hence

$$
\nu \oplus \mathcal{O}_{\mathbb{P}^{m-1}}(2)=\left[\mathcal{O}_{\mathbb{P}^{m-1}}(1)\right]^{m+1} .
$$

Thus

$$
c_{1}(\nu)=(m+1) h-2 h=(m-1) h,
$$

where $h$ is the hyperplane class of $\mathbb{P}^{m-1}$. Since $w_{2}(\nu)$ is the mod 2 reduction of $c_{1}(\nu)$, it follows that modulo 2

$$
S q^{2}\left(U_{\nu}\right)=\phi\left(w_{2}(\nu)\right)=(m-1) \phi(h) .
$$

Thus

$$
\begin{aligned}
S q^{2}(\eta) & =\alpha\left(S q^{2} U_{\nu}\right)=(m-1) \alpha(\phi(h)) \bmod 2 \\
& =(m-1) j_{!}(h)=(m-1) \xi j !(1)=(m-1) \xi \eta \bmod 2
\end{aligned}
$$


since $j^{*} \xi=h \bmod 2$ and $j$ ! is a $H^{*}\left(Q_{2 m-1}, \mathbb{Z}_{2}\right)$-module homomorphism. This proves the second formula. Since

$$
S q^{k}(a . b)=\sum_{i+j=k} S q^{i}(a) S q^{j}(b)
$$

(cf. (4), p. 91, (loc.cit.)), and $S q^{1} \equiv 0$ (odd cohomologies vanish), we have by the first two relations above

$$
\begin{aligned}
S q^{2}\left(\xi^{m-2} \eta\right) & =S q^{2}\left(\xi^{m-2}\right) \eta+\xi^{m-2} S q^{2}(\eta)=(2 m-3) \xi^{m-1} \eta \bmod 2 \\
& =\xi^{m-1} \eta
\end{aligned}
$$

which proves the third formula.

The lemma has been proved.

Proposition 16. Let $\mathcal{E}$ be a continuous complex vector bundle of any rank on $Q_{2 m-1}$ (where $m \geq 2$ ). In terms of the generators of $H^{2}, H^{4 m-4}$ and $H^{4 m-2}$ determined in Proposition 15, define its Chern numbers $c_{j} \in \mathbb{Z}$ by

$$
c_{2 m-1}(\mathcal{E})=c_{2 m-1}\left(x^{m-1} y\right) ; \quad c_{2 m-2}(\mathcal{E})=c_{2 m-2}\left(x^{m-2} y\right) ; \quad c_{1}(\mathcal{E})=c_{1} x
$$

Then we have

$$
c_{2 m-1}=c_{2 m-2}\left(c_{1}+1\right) \quad \bmod 2 .
$$

Proof. For a complex bundle $\mathcal{E}$, the Stiefel-Whitney class $w_{2 j}(\mathcal{E})$ is the mod 2 reduction of $c_{j}(\mathcal{E})$ (and odd Stiefel-Whitney classes vanish), so that

$$
w_{4 m-2}(\mathcal{E})=c_{2 m-1} \xi^{m-1} \eta ; w_{4 m-4}(\mathcal{E})=c_{2 m-2} \xi^{m-2} \eta ; w_{2}(\mathcal{E})=c_{1} \xi(\bmod 2),
$$

where $\xi$ and $\eta$ are the mod 2 reductions of $x$ and $y$ respectively, as in Corollary 11. We recall Wu's formula for Stiefel-Whitney classes of a real bundle (cf. Problem 8-B on p. 94, (loc.cit.)):

$$
S q^{2} w_{n}=w_{2} w_{n}+(2-n) w_{1} w_{n+1}+\frac{(2-n)(2-n-1)}{2} w_{0} w_{n+2} .
$$

For a complex vector bundle $\mathcal{E}, w_{1}(\mathcal{E})=0$, so applying the last formula for $n=4 m-4$, we have

$$
S q^{2}\left(w_{4 m-4}(\mathcal{E})\right)=w_{2}(\mathcal{E}) w_{4 m-4}(\mathcal{E})+w_{4 m-2}(\mathcal{E}) .
$$


Substituting from the first paragraph and Corollary 8, we have

$$
\begin{aligned}
S q^{2}\left(c_{2 m-2} \cdot \xi^{m-2} \eta\right) & =\left(c_{1} \xi\right) \cdot\left(c_{2 m-2} \xi^{m-2} \eta\right)+c_{2 m-1} \xi^{m-1} \eta \bmod 2 \\
c_{2 m-2}\left(\xi^{m-1} \eta\right) & =\left(c_{1} c_{2 m-2}+c_{2 m-1}\right) \xi^{m-1} \eta \bmod 2
\end{aligned}
$$

which implies the proposition.

Theorem 11. Let $m \geq 2$. Then a smooth quadric hypersurface $Q_{2 m-1} \subset \mathbb{P}^{2 m}$ does not have $\left(D_{c}\right)$.

Proof. The proof proceeds exactly as in the proof of Theorem 10. Since the normal bundle of $Q_{2 m-1}$ in $\mathbb{P}^{2 m}$ is $\mathcal{O}(2)$, the first Chern class of $Q_{2 m-1}$ is

$$
c_{1}\left(\tau_{Q_{2 m-1}}\right)=(2 m+1) x-2 x=(2 m-1) x .
$$

Thus

$$
w_{2}\left(\tau_{Q_{2 m-1}}\right)=\xi \in H^{2}\left(Q_{2 m-1}, \mathbb{Z}_{2}\right)
$$

Now let $\mathcal{E}$ be a complex vector bundle of complex rank $2 m-1$ on $Q_{2 m-1} \times Q_{2 m-1}$ realizing $\left(D_{c}\right)$. Then

$$
c_{1}(\mathcal{E})=a_{1}(x \times 1)+a_{2}(1 \times x) \in H^{2}\left(Q_{2 m-1} \times Q_{2 m-1}, \mathbb{Z}\right) .
$$

Since $\left(D_{c}\right)$ implies that $\delta^{*}(\mathcal{E}) \cong \tau_{Q_{2 m-1}}$, we have

$$
\delta^{*}\left(c_{1}(\mathcal{E})\right)=\left(a_{1}+a_{2}\right) x=c_{1}\left(\tau_{Q_{2 m-1}}\right)=(2 m-1) x,
$$

so that $a_{1}+a_{2} \equiv 1 \bmod 2$. The restrictions $\mathcal{E}_{i}, \quad i=1,2$ of $\mathcal{E}$ to the slices $Q_{2 m-1} \times\{p\}$ and $\{q\} \times Q_{2 m-1}$ respectively must have top Chern number $c_{2 m-1} \equiv$ $1 \bmod 2$ by Remark 6 . This implies, by Proposition 16, that $a_{1}$ and $a_{2}$ are both $\equiv 0 \bmod 2$. This contradicts the last paragraph. The theorem now follows.

Remark 11. It is not clear what happens for quadrics of even complex dimension. We note that $Q_{2}=\mathbb{C P}^{1} \times \mathbb{C P}^{1}$ and $Q_{4}=G_{2}\left(\mathbb{C}^{4}\right)$ both satisfy $\left(D_{c}\right)$ by Examples 2 and 3 .

We now make use of the topological Theorem 11, or rather its key input, Proposition 16, to obtain the following algebraic result.

Theorem 12. Let $X \subset \mathbb{P}^{2 n}$ be an odd dimensional smooth quadric hypersurface over an algebraically closed field $k$, with $n>1$. Then $X$ does not have (D). 
Proof. We claim that the congruence formula in Proposition 16 is valid for the algebraic Chern classes of any algebraic vector bundle, if we consider it as taking values in the Chow ring $C H^{*}(X) \otimes \mathbb{Z} / 2 \mathbb{Z}$. Assuming the claim, it follows that $X$ does not have the $\mathcal{O}_{X}(-r)$-point property for any odd $r>0$, exactly as in the complex topological case. Since $\omega_{X}=\mathcal{O}_{X}(-(2 n-1))$, we see from Corollary 6 that $X$ does not have (D).

Indeed, we will show that the congruence actually holds for Chern classes of virtual bundles, i.e. for the homogeneous components of any element in the image of the mod 2 total Chern class map

$$
K_{0}(X) \rightarrow C H^{*}(X) \otimes \mathbb{Z} / 2 \mathbb{Z} .
$$

We first observe that any smooth quadric $X \subset \mathbb{P}^{2 n}$ over an algebraically closed field $k$ is isomorphic to the quadric defined by

$$
\left\{x_{0}^{2}+\sum_{i=1}^{n} x_{i} x_{i+n}=0\right\} .
$$

This quadric contains the $i$-dimensional projective linear subspace

$$
L_{i}, \quad 0 \leq i \leq n-1,
$$

defined by

$$
x_{0}=0, x_{j}=x_{n+j}=0 \forall 1 \leq j<n-i, x_{j}=0 \forall j \geq n-i .
$$

Here, $L_{n-1} \cong \mathbb{P}^{n-1}$ is defined by $x_{0}=x_{1}=\ldots=x_{n}=0$, and $L_{0} \subset L_{1} \subset \cdots \subset$ $L_{n-1}$ is a maximal flag of projective linear subspaces. Let $H_{j} \subset X$ be a complete intersection with a general linear subspace of $\mathbb{P}^{2 n}$ codimension $j$.

The Grothendieck group $K_{0}(X)$ of algebraic vector bundles on $X$ is freely generated by the classes $\mathcal{O}_{H_{j}}, 0 \leq j \leq n-1$, and $\mathcal{O}_{L_{i}}, 0 \leq i \leq n-1$. Here $H_{0}=X$.

Similarly, the Chow ring of $X$ is freely generated, as an abelian group, by the classes of the irreducible varieties $H_{j}, 0 \leq j \leq n-1$ and $L_{i}, 0 \leq i \leq n-1$. Here $\left[H_{j}\right] \in C H^{j}(X)$, while $\left[L_{j}\right] \in C H_{j}(X)=C H^{2 n-1-j}(X)$.

If $x \in C H^{1}(X)$ is the class of the cycle $\left[H_{1}\right]$, and $y \in C H^{n}(X)$ the class of the cycle $\left[L_{n-1}\right]$, then the intersection product in $C H^{*}(X)$ is determined by the properties that the class of $\left[H_{j}\right]$ is just $x^{j}$, and the class of $\left[L_{j}\right]$ is $x^{n-j-1} y$. We also have relations $y^{2}=0$ and $x^{n}=2 y$. 
For $k=\mathbb{C}$, this is exactly the presentation for the integral cohomology ring $H^{*}(X, \mathbb{Z})$ given in Proposition 15 (with $m$ in place of $n$ ). In fact, the cycle class map

$$
C H^{*}(X) \rightarrow H^{*}(X, \mathbb{Z})
$$

induces an isomorphism of rings, where the algebraic generators $x, y$ above map to the corresponding topological generators of Proposition 15.

In a similar fashion, if $K_{\text {top }}(X)$ denotes the topological $K$-group of complex vector bundles on $X$, one knows that the natural map $K_{0}(X) \rightarrow K_{\text {top }}(X)$ is an isomorphism of rings. Further, the algebraic and topological Chern class maps are compatible. Hence, an element of $C H^{*}(X)$ lies in the image of the algebraic total Chern class map precisely when the corresponding element in $H^{*}(X, \mathbb{Z})$ is in the image of the topological total Chern class map. Note that, at the topological level, any element of $K_{t o p}(X)$ is of the form $[\mathcal{E}]-m$ for some integer $m$, where $\mathcal{E}$ is a complex topological vector bundle on $X$. Thus, the image of the total topological Chern class map coincides with the set of total Chern classes of complex topological vector bundles. In particular, at the topological level, the congruence in Proposition 16 is valid for the total Chern class of any element on $K_{t o p}(X)$. Hence this congruence is valid for the corresponding algebraic total Chern class image of $K_{0}(X)$ in $C H^{*}(X)$.

We now argue that, in fact, the image of the algebraic total Chern class map is the same, for any smooth quadric $X \subset \mathbb{P}^{2 n}$ over an algebraically closed field $k$, where we identify the Chow rings of all such quadrics using the explicit presentation. This is because the formulas for the Chern classes of the generators $\left[\mathcal{O}_{H_{j}}\right]$ and $\left[\mathcal{O}_{L_{i}}\right]$ of $K_{0}(X)$, expressed as polynomials over $\mathbb{Z}$ in the generators $x, y$ for the Chow ring, are independent of the field $k$. This is clear for the $H_{j}$ since these are classes pulled back from projective space, and Chern classes are functorial under pullback; these classes are certain polynomials in $x$. For the classes of $L_{i}$, we may regard these as in the image of the push-forward map $K_{0}\left(L_{n-1}\right) \rightarrow K_{0}(X)$. The push-forward map on the graded Chow group is also defined, identifying $C H^{j}\left(L_{n-1}\right) \cong \mathbb{Z}$ with $C H^{n+j}(X) \cong \mathbb{Z}$, where the cycle $\left[L_{j}\right]$ is a generator of either group.

Now, the universal nature of the Chern class formulas for the sheaves $\mathcal{O}_{L_{i}}$ follows from the theorem of Riemann-Roch "without denominators" ([9], Theorem 15.3) for this push-forward map. 
Thus, the truth of the congruences of Proposition 16 in the topological case, hence the complex algebraic case, implies the same congruences hold over any algebraically closed field.

Remark 12. In fact the Riemann-Roch formula in [9], Theorem 15.3 allows us, in principle, to consider giving a direct algebraic proof of Proposition 16 for elements in the image of the algebraic total Chern class map, by actually determining explicitly the image of the total Chern class map itself. This would also prove the result in the topological case as well, by reversing the above argument, and would in some way "explain" it, independent of any identities or properties of Steenrod squares, for example. However, we were so far unable to make the direct computation. In principle, such a direct approach might also shed light on the case of even dimensional quadrics, and perhaps other cases. We conjecture that a smooth quadric hypersurface $Q_{n}$ in the projective $(n+1)$-space (over an algebraically closed field) has (D) if and only if $n=1,2$ or 4 .

Note In a recent letter [6], O. Debarre has informed us that he proved (D) for Jacobians of smooth projective connected curves, and found counterexamples to (D) for general abelian varieties of dimension greater than 2 .

Acknowledgments The first two authors started cooperation on this paper during the Conference "Antalya Algebra Days 2005", and they thank the organizers for creating this opportunity.

P. Pragacz thanks Professor Friedrich Hirzebruch for his interest towards the "diagonal property" as well as for helpful comments received before and during the author's stay at the MPIM in Bonn in 2006.

V. Pati is grateful to the other two authors for inviting him on board this interesting investigation, and valuable insights. Also to Jishnu Biswas for educating him on complete intersections and pointing out the relevant references, and Shreedhar Inamdar for useful discussions and encouragement.

The authors also thank A. Ozgur Kisisel for pointing out an error in a former version of the paper.

\section{REFERENCES}

[1] C. Bănică, M. Putinar, On Complex Vector Bundles on Projective Threefolds, Inv. Math. 88 (1987), 427-438. 
[2] W. Barth, C. Peters, A. Van de Ven, Compact complex surfaces, Ergebnisse Math. (3) 4, Springer-Verlag, Berlin, 1984.

[3] A. Beauville, Surfaces algébriques complexes, Astérisque 54, Société Math. de France, Paris, 1978.

[4] M. Brion, Lectures on geometry of flag varieties, in: "Topics in cohomological studies of algebraic varieties", Trends in Mathematics, Birkäuser, Basel 2005, 33-85.

[5] S. D. Cutkosky, On Fano 3-folds, Manuscripta Math. 64 (1989) 189-204.

[6] O. Debarre, Letter to the authors, 20.12.2006.

[7] L. Ein, I. Sols, Stable vector bundles on quadric hypersurfaces, Nagoya Math. J. 96 (1984), 11-22.

[8] D. Eisenbud, S. Popescu, Ch. Walter, Lagrangian subbundles and codimension 3 subcanonical schemes, Duke Math. J. 107 (2001), 427-467.

[9] W. Fulton, Intersection theory, 2nd Edition, Ergebnisse Math. (3) 2, Springer-Verlag, Berlin, 1998.

[10] W. Fulton, Flags, Schubert polynomials, degeneracy loci, and determinantal formulas, Duke. Math. J. 65 (1992), 381-420.

[11] W. Fulton, P. Pragacz, Schubert varieties and degeneracy loci, Lecture Notes in Math. 1689, Springer-Verlag, Berlin, 1998.

[12] W. Graham, The class of the diagonal in flag bundles, J. Diff. Geom. 45 (1997), 471-487.

[13] Ph. Griffiths, J. Harris, Principles of algebraic geometry, Pure and Applied Math., Wiley-Interscience, New York, 1978.

[14] A. Grothendieck, La théorie des classes de Chern, Bull. Soc. Math. France 86 (1958), $137-154$.

[15] R. Hartshorne, Algebraic Geometry, Graduate Texts in Math. 52, Springer-Verlag, Berlin, 1977.

[16] R. Hartshorne, Ample subvarieties of algebraic varieties, Lecture Notes in Math. 156, Springer-Verlag, Berlin, 1970.

[17] F. Hirzebruch, Topological methods in algebraic geometry, Second corrected printing of the 3rd edition, Grundlehren Math. 131, Springer-Verlag, Berlin, 1978.

[18] V. A. Iskovskih, Fano threefolds, I, II, Izv. Akad. Nauk SSSR Ser. Mat. 41 (1977), no. 3, 516-562, 717, and 41 (1978), no. 3, 506-549.

[19] M. M. Kapranov, On the derived categories of coherent sheaves on some homogeneous spaces, Inv. Math. 92 (1988), 479-508.

[20] A. Lascoux, M-P. Schützenberger, Polynômes de Schubert, C. R. Acad. Sci. Paris 294 (1982), 447-450.

[21] M. Levine, V. Srinivas, J. Weyman, K-theory of twisted Grassmannians, K-theory 3 (1989), 99-121. 
[22] J. Milnor, Some consequences of a theorem of Bott, Ann. of Math. 68 (1958), 444449.

[23] J. Milnor, J. Stasheff, Characteristic classes, Annals of Math. Studies 76, Princeton Univ. Press, 1974.

[24] D. Mumford, Abelian varieties, Tata Inst. Studies in Math. 5, Oxford Univ. Press, 1970.

[25] D. Mumford, An algebraic surface with $K$ ample, $\left(K^{2}\right)=9, p_{g}=q=0$, Amer. J. Math. 101 (1979), 233-244.

[26] N. Mohan Kumar, M. P. Murthy, Algebraic cycles and vector bundles over affine three-folds, Ann. of Math. 116 (1982), 579-591.

[27] M. P. Murthy, Zero cycles and projective modules, Ann. of Math. 140 (1994), 405434.

[28] P. Pragacz, Symmetric polynomials and divided differences in formulas of intersection theory, in: Parameter Spaces, Banach Center Publications 36, Warszawa 1996, 125-177.

[29] P. Pragacz, J. Ratajski, Formulas for Lagrangian and orthogonal degeneracy loci; $\tilde{Q}$-polynomial approach, Compositio Math. 107 (1997), 11-87.

[30] G. Prasad, S-K. Yeung, Fake projective planes, math.AG/0512115.

[31] J-P. Serre, Sur les modules projectives, Séminaire Dubreil-Pisot (1960/61), Exposé 2.

[32] J-P. Serre, Algèbre locale, multiplicités, Lecture Notes in Math. 11, Springer-Verlag, Berlin, 1975.

[33] N. I. Shepherd-Barron, Fano threefolds in positive characteristic, Compositio Math. 105 (1997), 237-265.

Piotr Pragacz

Institute of Mathematics of Polish Academy of Sciences

Śniadeckich 8, 00-956 Warszawa, Poland

E-mail: P.Pragacz@impan.gov.pl

Vasudevan Srinivas

School of Mathematics, Tata Institute of Fundamental Research

Homi Bhabha Road, Colaba, Mumbai-400005, India

E-mail: srinivas@math.tifr.res.in

Vishwambhar Pati

Stat-Math Unit, Indian Statistical Institute

RV College Post, 8th Mile, Mysore Road, Bangalore-560059, India

E-mail: pati@isibang.ac.in 